\title{
A NUMERICAL AND EXPERIMENTAL STUDY OF A NEW DESIGN OF CLOSED DYANMIC RESPIRATION CHAMBER
}

\author{
Ahmed Al Makky. ${ }^{1}$, A. Alaswad ${ }^{2}$, D. Gibbson ${ }^{3}$, S. Song ${ }^{4}$ and A. G. Olabi ${ }^{5}$
}

1. School of Engineering, University of the West of Scotland, Paisley: email: Ahmed.AlMakky@uws.ac.uk

2. School of Engineering and the Built Environment, Birmingham City University, Birmingham, England; email: Abed.Alaswad@bcu.ac.uk

3. Scottish Universities Physics Alliance, Institute of Thin Films, Sensors \& Imaging email: Des.Gibson@uws.ac.uk

4. Scottish Universities Physics Alliance, Institute of Thin Films, Sensors \& Imaging email: Shigeng.Song@uws.ac.uk

5. School of Engineering, University of the West of Scotland, Paisley; email: Abdul.Olabi@uws.ac.uk

\section{Abstract}

Carbon dioxide soil efflux modelling in closed dynamic respiration chambers is a challenging task. This is attributed on many occasions to the very small concentrations of carbon dioxide being transported between soil and the atmosphere. This paper describes a portable device which was made exclusively to accurately measure carbon dioxide efflux from soil locations. The blowing fan creates a forced convective flow to occur in the chamber making the KEpsilon turbulence model a necessity to model the occurring flow in the respiration chamber gas domain. Furthermore the Darcy model is applied on the porous domain to model the flow pattern within the soil. The measurement process was achieved through measuring carbon dioxide concentration, temperature and relative humidity inside the chamber in relation to time. Simulation and experimental data is obtained using ANSYS and MATLAB. A significant agreement between the experimental and numerical results was achieved.

Keywords: Carbon Dioxide, CFD, K-Epslion, Porous Media, Dynamic Chambers, Global Warming 
A

A

$A_{n}$

B

C

$\mathrm{C}(\mathrm{t})$

$\widetilde{C(t)}$

$\mathrm{D}_{\mathrm{n}}$

$D_{\text {total }}$

$\mathrm{d}_{\mathrm{av}}$

$\mathrm{H}(\mathrm{z})$

K

$\mathrm{K}^{\mathrm{ij}}$
Sample area cross section

Infinitesimal planar control surface

Area of a single pore

Body force vector

Carbon dioxide constants in Sutherland

equation

Carbon dioxide concentration as a

function of time

Carbon dioxide filtered concentration as

a function of time

Total Integrated area for permeability

function

Total area of pores

The average pore diameter for a

segment of pore sizes

Concentration Filter function

Soil permeability

Area porosity tensor 


\begin{tabular}{|c|c|}
\hline $\mathrm{K}_{\text {perm }}$ & Permeability \\
\hline $\mathrm{K}_{\text {loss }}$ & Empirical loss coefficient \\
\hline $\mathrm{P}_{\mathrm{k}}$ & Turbulence production \\
\hline$\grave{p}$ & modified pressure \\
\hline $\mathrm{R}^{\mathrm{ij}}$ & Resistance to flow in the porous \\
\hline & medium \\
\hline$r_{\text {sand }}$ & Sand grain diameter \\
\hline $\mathrm{r}_{\text {silt }}$ & Silt grain diameter \\
\hline $\mathrm{r}_{\text {clay }}$ & Clay grain diameter \\
\hline $\mathrm{T}$ & Instance of time \\
\hline $\mathbf{U}$ & Vecloity vector field \\
\hline V & Studied volume of soil \\
\hline \multirow[t]{2}{*}{ V } & The volume available to flow in an \\
\hline & infinitesimal control cell surrounding \\
\hline
\end{tabular}

34 - Greek Symbols
$\Gamma_{\mathrm{e}}$
Effective thermal diffusivity
$\Gamma$
volume porosity 


$\begin{array}{ll}\Theta & \text { Volumetric water content } \\ M & \text { Air dynamic viscosity } \\ \mu_{\mathrm{e}} & \text { Effective viscosity } \\ \mathrm{P} & \text { Air density }\end{array}$

\section{Introduction}

Soil can be defined as a complex system, consisting of a mixture of organic and mineral particles, soil solution and air, resulting from the interaction between biotic and abiotic factors; it is the medium in which plants acquire water and nutrients through their roots system. It is known by scientists that one of the physical properties of carbon dioxide contained in the atmosphere is that it reflects heat back to the earth's surface. Consequently gradually the earth's atmosphere traps more heat. Respiration chambers can be used to quantify the soil efflux whereby they come in different shapes and sizes this depends on their application of use. They are composed of two main parts; namely the chamber shell and the gas sensor. To quantify the amount of produced carbon dioxide at one location, an enclosed cavity or space like a chamber is used. An efflux is flowing out or forth from a porous medium (Soil) which for our case of concern is carbon dioxide. Carbon dioxide gas in the soil is produced due to the biological activity in the soil domain. This method was first proposed by Henrik Lundegardh in the form of the respiration bell [1]. In the general context, studying respiration chambers can give scientists some insight to how fertile the studied site is. That is by measuring the rate of carbon dioxide produced for a certain site of concern in order to predict its impact on global warming issues [2]. Consequently with the increase of carbon dioxide concentrations in the atmosphere, 
earth responds to it [3]. Climate change is one of the most critical challenges that are facing the mankind, and it is well related to the greenhouse emissions which help trapping heat and making the earth warmer that affect directly weather patterns, people, plants and animals.

Greenhouse fluxes measurement between the soil and the atmosphere is of a great importance to help to understand the biochemical parameters effects on the global warming issue. This has lead scientists to use numerical nonlinear models to predict future concentrations of carbon dioxide in the atmosphere [4]. Subsequently others used more sophisticated models such as the dynamic global vegetation model [5] as shown in Figure 1.
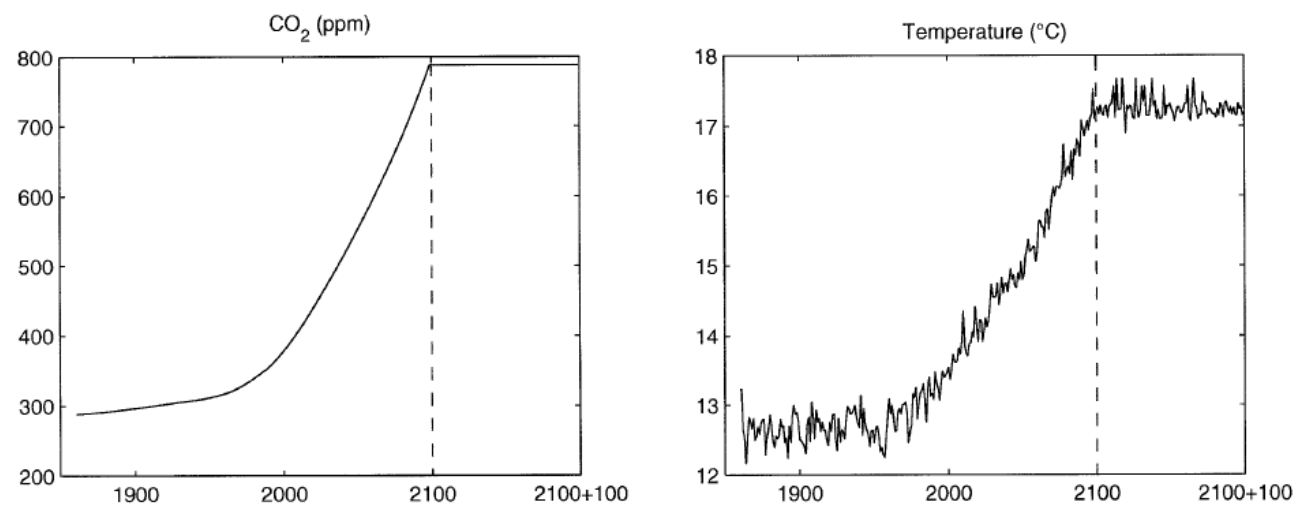

Figure 1: IPCC IS92a projections of atmospheric CO2 concentration and the HadCM2 SUL climate model simulations of temperature over land (excluding Antarctica).

The used chamber methods have been surveyed in [6] where by it showed that new methods were proposed since the early 80s with growing interest in the global warming issue, Kyoto protocol. Scientists using these chambers can quantify the soil site carbon budget [7].

The rational for this study is to interpret and quantify for a specific location how soil produced carbon dioxide contributes to the greenhouse effect. This is done by using a designed respiration chamber by our research group. The main focus of this study is to use CFD numerical models to show how they can assist in making the right dynamic chamber designs to get the right measured fluxes representing the biological activity of location. This helps in 
74 finding the right locations to install the gas sensors within the chamber and also to ensure through mixing for the gas mixture. The process is firstly conducted using CAD and secondly using CFD design optimization. The chamber uses a sampling tube connected with the sensor hence it takes samples at all the inner chamber elevations furthermore it relies on the convective flow produced by the fan to get the correct measurements instead of using a gas sensor that sucks the gas sample and takes several seconds to analyse it. The selection of the blowing fan location is also selected to draw out the carbon dioxide within the soil layer and at the same time not to cause any disturbance to the biological activity in the soil as for cooling or increasing water evaporation rates. A significant agreement between the experimental and numerical results was achieved.

\section{Respiration Chambers}

Scientists know that no ideal experimental chamber exists [8], therefore the aim is always to reduce measured errors. That is due to the great spatial variability in soil emissions, and to the fact that the quantification of these emissions is complicated by the high spatial variability exhibited by many microbial processes [9]. Respiration chambers are produced either privately for research groups or by commercial companies. The transparent chamber is intended to be used to measure total flux from a specific location, it is automated to ventilate the chamber, while the none-transparent one is the total flux excluding the flux resulting from photosynthesis process. The top hat type chamber is used for a quick site deployment where ventilation is conducted manually, mostly intended for soil flux measurements. Different types of chambers are available depending on the intended efflux quantity to be measured as shown in Figure 2. The figure presents an example of chambers produced by the Li-Cor Inc Company with the named different parts. There are four types of chambers characterised according to their operational mode these are closed dynamic, open dynamic, closed static and open static chambers. 


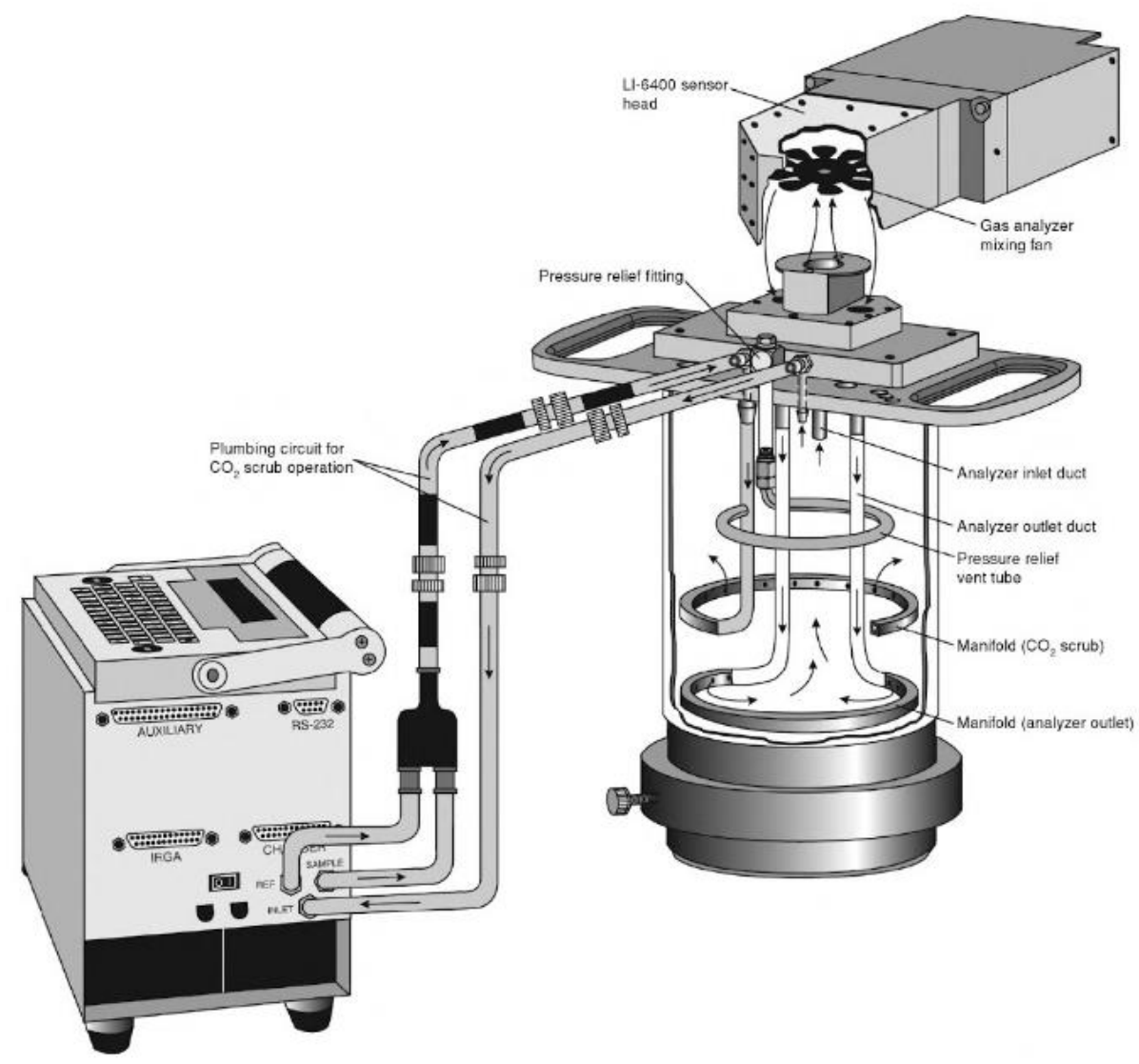

Figure 2: An example of an air circulation closed dynamic chamber made by Li-Cor Inc model (Li-cor 6400-09)[10] .

\subsection{Methods of efflux Measurements}

Scientists have an option to choose from several methods for measuring soil carbon dioxide

efflux. These methods can be summarised into four ones, starting with the chamber soda lime

[11] or what is called sometimes by alkali solution method which absorbs the respired carbon dioxide from the soil, it is an easy and cheap method to apply. The second method is by using the soil carbon dioxide gradient system method [12], generally this method is much complicated and not easy to setup. It requires the insertion of the gas sensors inside the soil layers of the studied location. This action disturbs the location integrity in addition to that gas sensors are expensive. The third method is the Eddy covariance method, sometimes referred to as micrometeorological method [13], the positive point about it that it doesn't disturb the

112 location of study, because all the necessary sensors are attached to a tower overhead the location of study. This method can be regarded as an expensive method and represents a more 
attractive option to measure carbon dioxide emissions from spacious locations such as farmlands considering that plant community canopy emissions can also be studied taking advantage of the tower setup. Finally the fourth method is by using respiration chambers that use gas sensors such as infra-red gas sensors. These chambers are easy to use and setup, they do introduce disturbance to the soil surface upper crust when using the chamber fixture method such as the clamp method. However the cost of having several methods to choose from is the large difference in accuracy, spatial and temporal resolution, and applicability. Therefore some kind of compromise has to be made in the choice of (accuracy and resolution) and feasibility (applicability and cost) [14]. That is why researchers have used different efflux measurement methods and cross calibration functions to overcome these uncertainties [15]. The developed methods can be used to validate and calibrate other classical methods used in carbon dioxide measurements. The problem of under or over prediction of measured efflux, as described in $[16]$ is due to external turbulences.

\subsection{What is the drive behind using closed dynamic chambers?}

The main challenge of measuring carbon dioxide concentrations is acquiring instantaneous samples every second of time. The setback in using static chambers is that the diffusion time crust layers and inside the chamber requires longer periods of measurements. Hence relying on diffusion alone for mass transport is not practical time wise. Consequently the dynamic chamber method is used for the reason that it relies on forced mass transport for the carbon dioxide species. This is achieved through the use of a blowing fan inside the chamber. That would decrease the required time for onsite deployment and sampling. Whereas as you move down in depth from the soil surface through the soil layers mass diffusion becomes the dominant factor of species transport. Hence we are interested in drawing out the gas mixture of air and carbon dioxide for the specified 6 minutes of the experiment from the soil $\mathrm{O}, \mathrm{A}$ and 
B horizons. Due to that the measurement period is 6 minutes the blowing fan intention is to create the suction affect to take out the stored carbon dioxide [17]. Production velocities of carbon dioxide within the soil layers are in the order of $10^{-5} \mathrm{~m} / \mathrm{s}$ to $10^{-7} \mathrm{~m} / \mathrm{s}$. So by sucking out all the carbon dioxide stored in the $\mathrm{O}$ and $\mathrm{A}$ horizons volume the biological active has no time to replenish the complete mass taken out. Hence by measuring it in 6 minutes biological activity will not have enough time to produce additional carbon dioxide. This study is focused mainly for closed dynamic chambers operational mode and can also be applied to static chamber mode hence no venting tube is required.

\section{The Designed Chamber}

A chamber has been designed and made at the University of the West of Scotland (UWS), sensors were fit inside it properly, which allowed experimental results to be attained. Consequently, CFD numerical simulations using ANSYS (to model the fluid flow) were conducted, and further experimental studies were carried out. When the chamber was designed, these assumptions where made:

1- The chamber can operate in two modes the first is the steady mode (switched off fan) relying on diffusive mass transport. The second mode is the unsteady mode (switched on fan) relying on flow convection for mas transport, at the unsteady state the airflow should sweep over the entire covered soil surface.

2- The gas efflux should be of uniform magnitude over the covered surface by the chamber. The pattern of the airflow in the chamber should be relatively uniform in speed across the swept soil surface. This is to create the necessary suction pressure to draw out the carbon dioxide gas species from the top soil layer.

3- The fan inflow and outflow should ensure that a well-mixed air $/ \mathrm{CO}_{2}$ mixture is circulated inside the chamber. 
4- The diffusive flux is dominant in the steady state of operation likewise the advective flow is negligible, on the contrary for the unsteady state the case is vice versa.

5- The pressure difference between the inside and outside of the chamber should be kept to the minimum that is through using the chamber installation base with the soil.

6- The material for chamber fabrication should be strong to avoid possible structural deformation under field conditions. A deformed chamber body may cause leakage in the system and produce errors.

7- The outside surface of the chamber should be able to reflect partially solar radiation [17]. Considering that each type of used chamber shell material has a predefined transmissivity property.

Based on these assumptions, a CAD model was made as shown in Figure 3 this will be used for calculating the steady-state flux. All respiration chambers have the general chamber shape configuration the design contribution is evident when a comparison is conducted between Figure 2 and Figure 3. For example a noted design difference is that the LICOR designs have no circulation fan within them blowing in a perpendicular manner on the soil surface. The built model is shown on Figure 4 installed on the grass land location where the experimental measurements were taken. A general description of the experimental apparatus is that it is a cylindrical transparent plastic (perspex) chamber having a height of $0.5125 \mathrm{~m}$ and a diameter of $0.38 \mathrm{~m}$. These dimensions create a chamber footprint of $0.113 \mathrm{~m}^{2}$ with an internal volume of $0.06 \mathrm{~m}^{3}$. It has one cap cover at the top of the chamber to allow the proper fitting of the blowing fan configuration in addition to providing flexibility of distance control of the convective flow intensity on the soil surface. The chamber covers a circular area of the soil. During the experiment the system is placed over the soil surface at ambient temperature. 


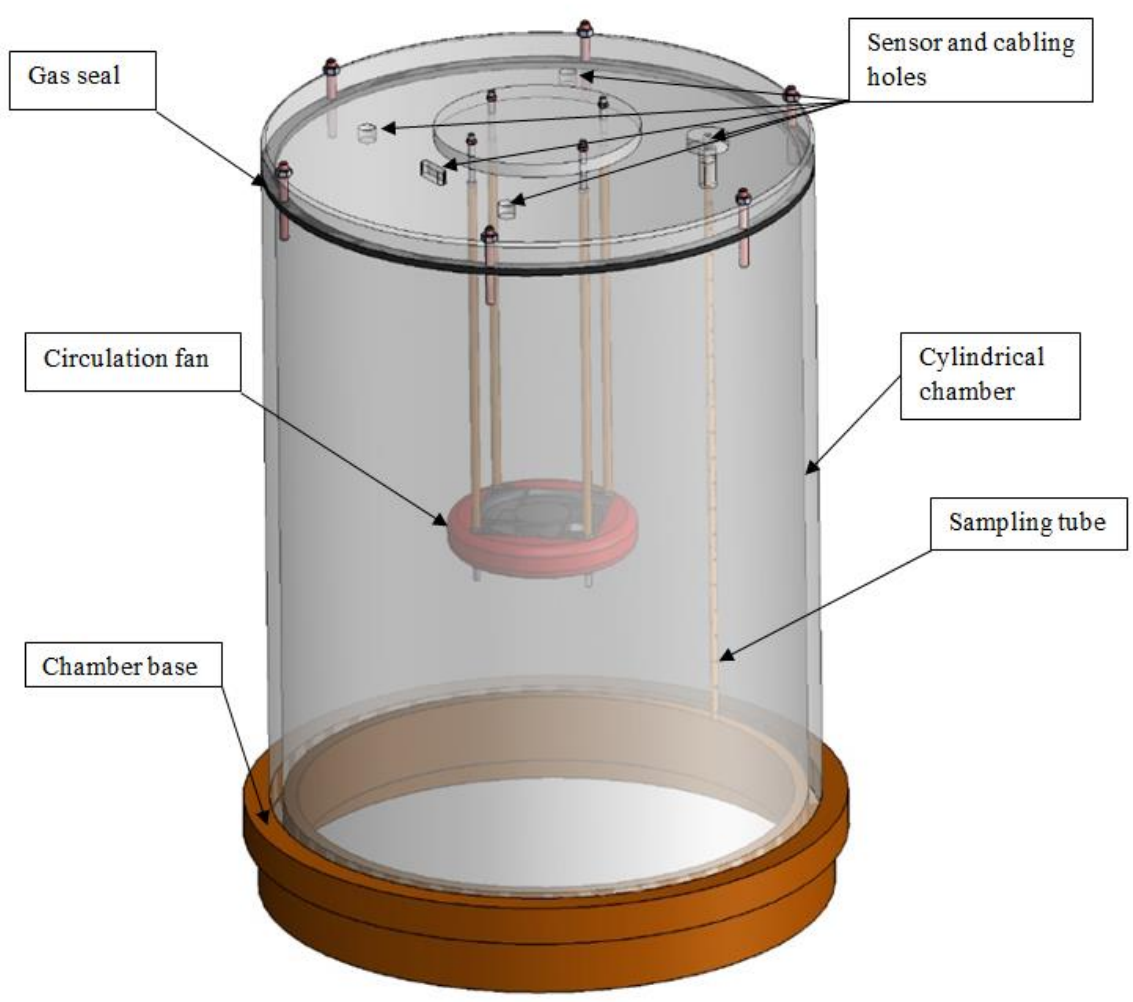

Figure 3: The agreed upon CAD design for the chamber to use for the study.

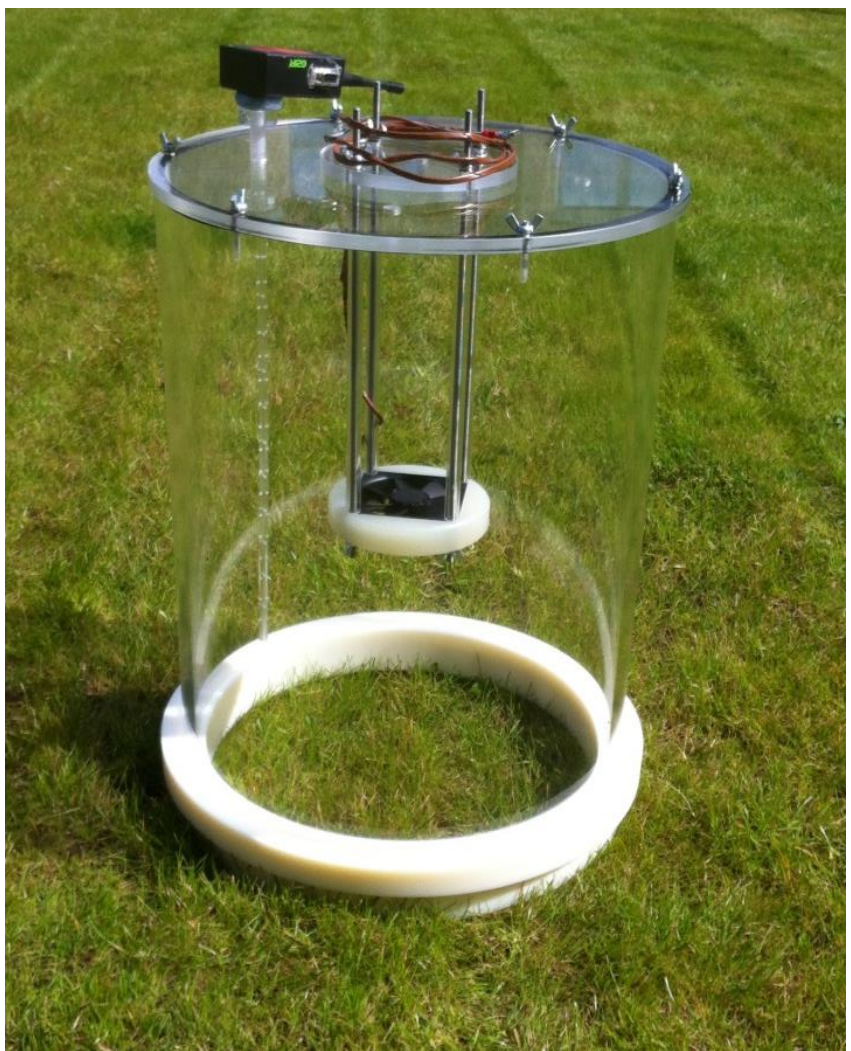

Figure 4: The used designed chamber at the University of the West of Scotland, located on the grass land site. 
Chamber venting happens by turning over the chamber for ventilation after the measurement then switching the fan on to blow out the accumulated carbon dioxide from within the chamber volume. The reason for not selecting a parallel to the soil blowing fan is that blowing jet hitting the chamber wall would cause a none necessary rise in internal pressure causing gas leakage out of the chamber. Hence the fans distance was thoroughly selected to produce a pressure of 0.7 Pa. This value is equivalent to blowing winds in the range from $1 \mathrm{~m} / \mathrm{s}$ to $5 \mathrm{~m} / \mathrm{s}$ encountered on the site location of interest this idea is discussed in [17].

\subsection{The Sensing Box}

The sensing box as shown in Figure 5 is composed of the sensors, shown in Table 1, that are connected to a microcontroller. The first one is the temperature sensor, relative humidity, Dew point and light intensity. The second is the chemical species sensor which uses the nondispersive infrared gas analyzer which measures the carbon dioxide concentrations. The sensor can work on battery mode while on location or can be plugged to the electrical mains for Lab tests or for battery charging. The sensor box is fitted with the wireless antenna to transfer data to the wireless rotor connected to the laptop. The sensing box is fitted to a sampling tube which collects the data from within the chamber; the sampling tube has side holes to insure that sampling is taken at the different elevations inside the chamber as shown in Figure 4. By using this method a homogenous gas mixture is sampled. Meaning that better resolution concentration measurement in relation to time is achieved. A precise calibration was made before the experiments this was through measuring the standard atmospheric concentration of carbon dioxide. The sensors sampling period was modified from 30 seconds to 5 seconds. For the reason that diffusion time required 30 seconds for gas species to get to the tip of the sensor in a static chamber case. The challenge was resolved through using a convective flow pattern with the chamber. Pumping samples out of the chamber wasn't used because some gas sensors 
217 dump the analysed gas sample into the outer environment of the chamber. The purpose of using 218 closed chambers is to accumulate carbon dioxide concentrations within the gas volume to 219 capture the exponential concentration curve. Gas sensors that use gas sampling technique 220 similar to the syringe concept are not continuous because they require several seconds for the 221 gas sample to be pumped out of the chamber and a further several seconds to conduct sample analysis. Due to using dynamic chamber and a 6 minute time measurement the issues of condensation is overcome basically because a homogenous temperature heat field is created within the chamber. The used sensor properties are summarized in Table 1, while the experimental setup and data collection steps for the dynamic chamber experiment are summarised in Figure 6.

227 Table 1: Technical details of the non-dispersive infrared gas analyzer.

\begin{tabular}{|l|l|}
\hline Accuracy: & $+/-50 \mathrm{ppm}$, or $3 \%$ of FS \\
\hline Response time & $<10 \mathrm{~s}$ \\
\hline Range & $0-5000 \mathrm{ppm}$ \\
\hline Working Environment & $0 \sim 50^{\circ} \mathrm{C}, 0 \sim 95 \% \mathrm{RH}$ (No water condense) \\
\hline Storage temperature & $-20 \sim 80^{\circ} \mathrm{C}$ \\
\hline Power & $3.5 \mathrm{~mW}$ \\
\hline
\end{tabular}




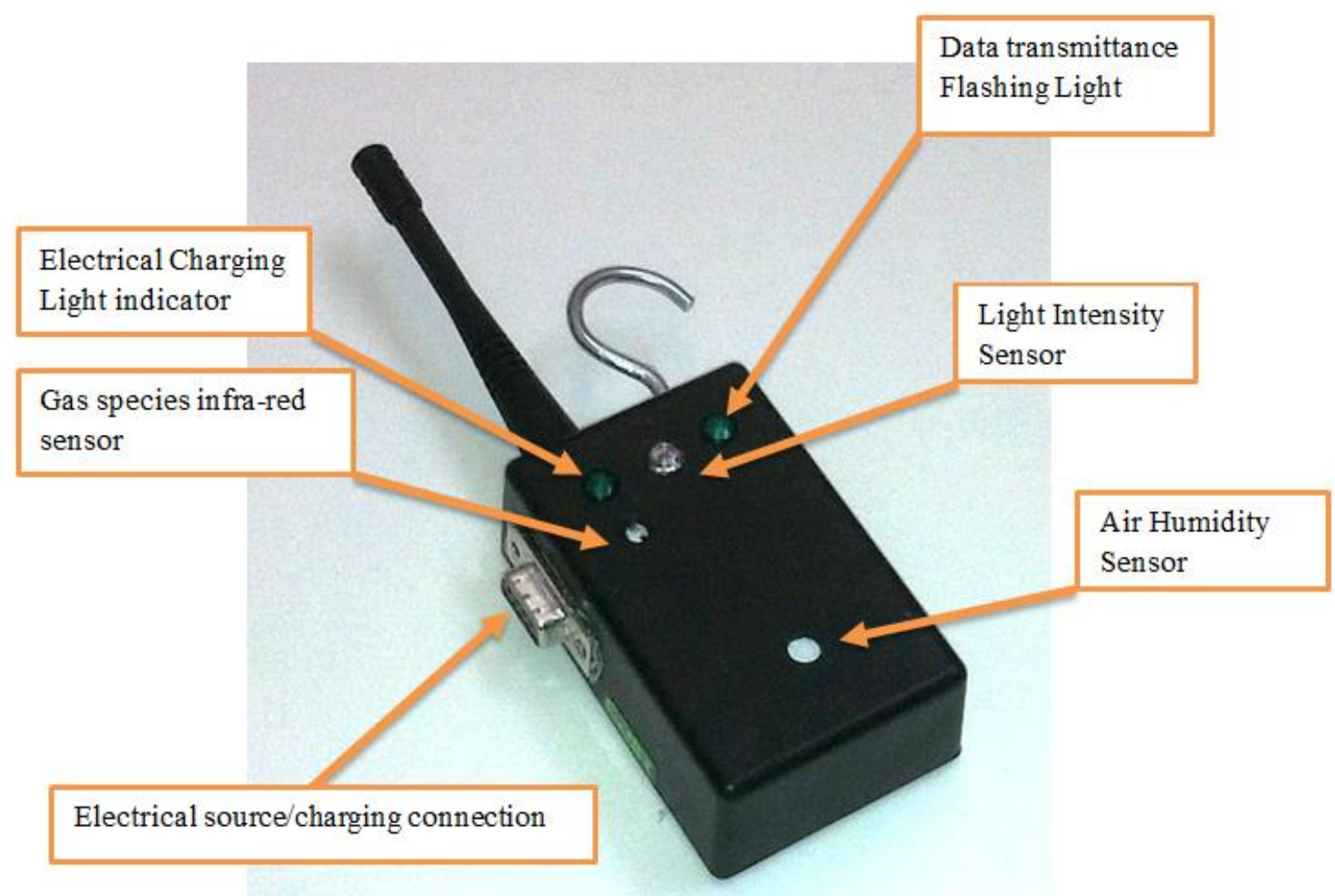

Figure 5: The used sensor box which has the main purpose to measure carbon dioxide concentrations. 


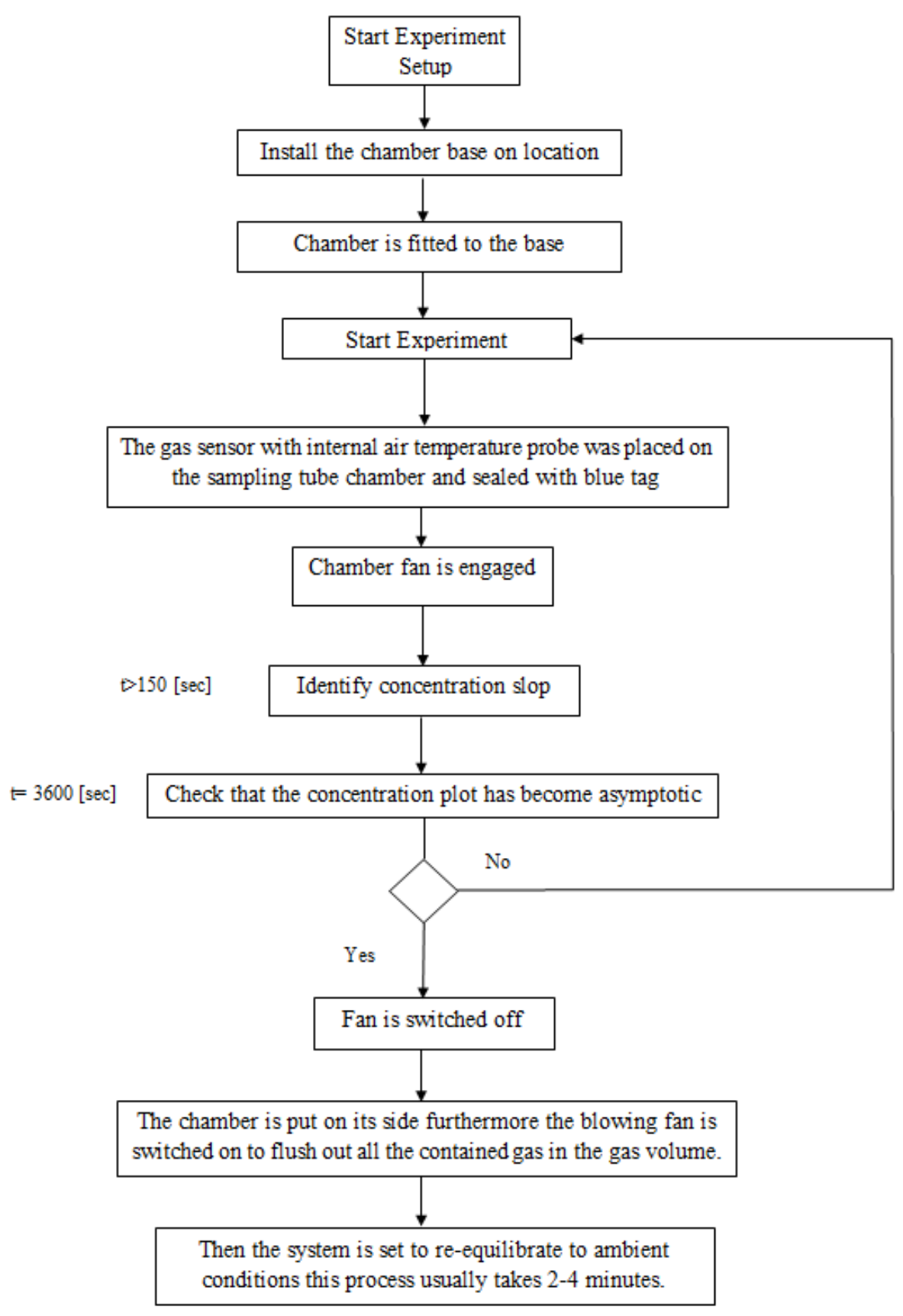

Then the system is set to re-equilibrate to ambien

235 Figure 6: The flow chart showing the experimental setup steps and data collection for the 236 dynamic chamber experiment.

\subsection{Location of Study}

239 Measurements were taken in Paisley which is a town located in the western part of Glasgow city. The terrain is moderately hilly near to the location as seen in Figure 7 were the location 
242 the relation of location height with air density. Its importance becomes apparent when

243 calculating chamber internal pressure further more in calculating chamber air mass and rate of

244 air volume recirculation in relation to time for the chamber gas volume.

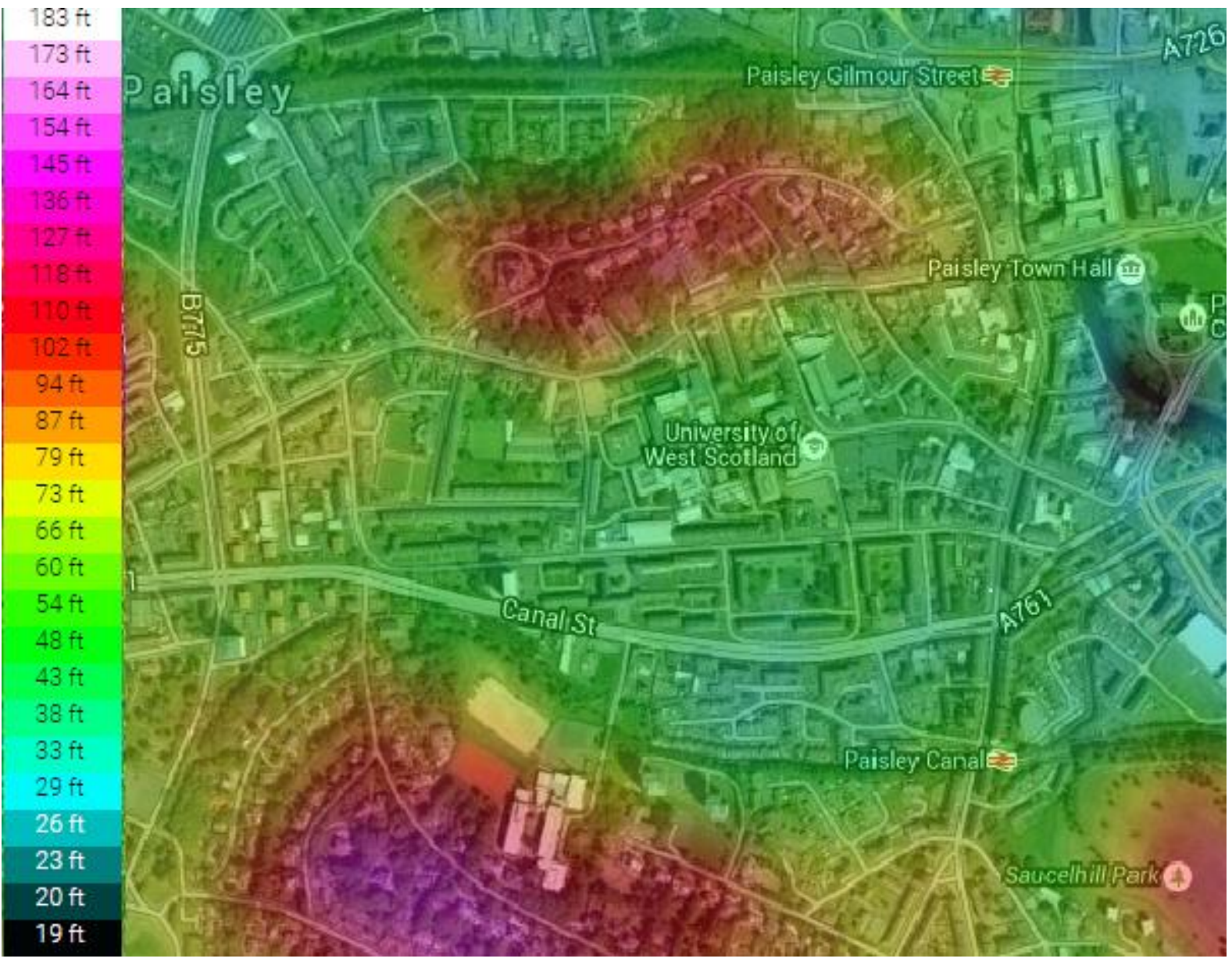

Figure 7: Topographic map of the location of study, the site is located on University of West colour range.

The study area is located on the prospect location tract $\left(55^{0} .50^{`} \mathrm{~N}, 4^{0} .26^{`} \mathrm{~W}\right)[18]$ as shown in

Figure 8. The selected site is a managed grassland located in the University of West Scotland

The main characteristic of the grassland site as mentioned by [19] is that it has high fertility. 
254 A managed grass land is regarded as an ideal case for a studied site location hence Figure 4is used as visual proof of location of study.

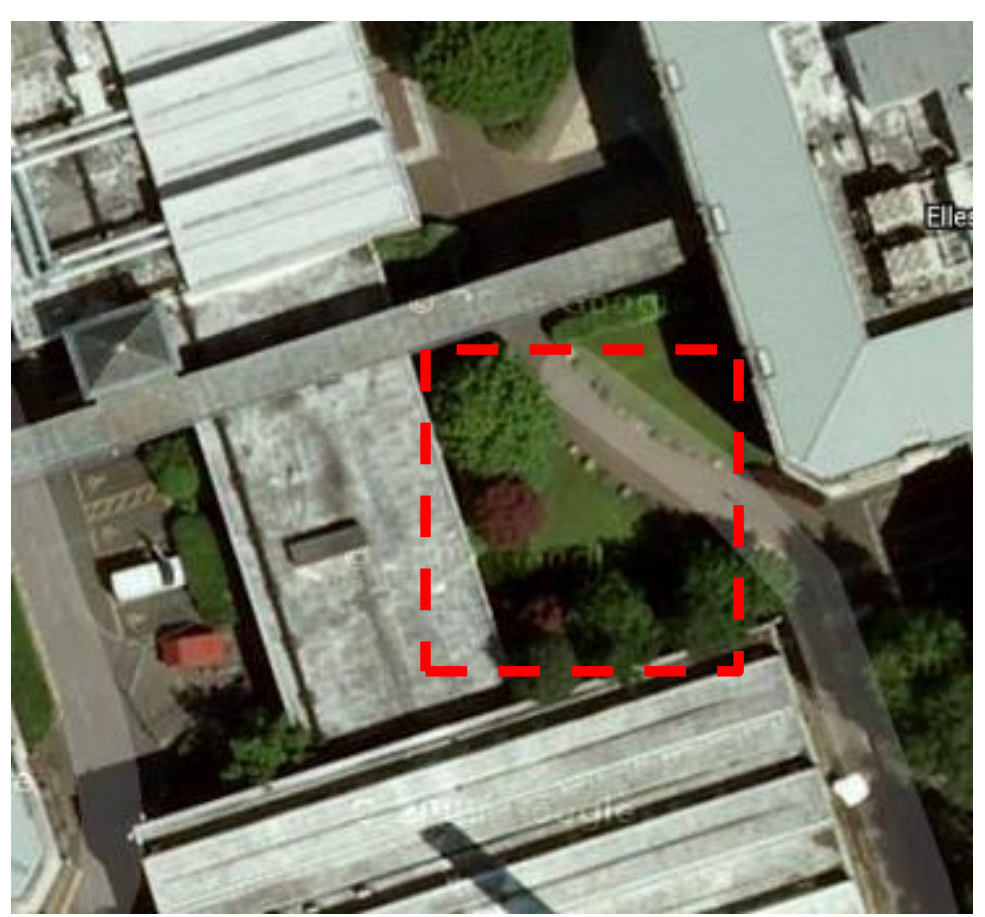

Figure 8: The managed grass land location of study at the UWS paisley campus.

The chamber base is not permanently installed into to the location it is pushed smoothly into the soil surface. But the main emphasis is to preserve the soil surface integrity as evident in Figure 4. As will be evident in section 3.3 that a managed grass land location can be identified on the soil texture plot shown in Figure 9 hence its porosity and permeability values can be identified which are used as CFD simulation input values.

From experiments it was evident that for a static chamber data from the university weather station is very essential for the reason that on hourly bases big temperature difference becomes evident. This is attributed for that diffusion time is long especially for a chamber with a relatively high headspace. While an average day time temperature measurement can be satisfactory for a dynamic chamber study this is because the measurement period of 6 minutes. This is for a case for acquired data from the met office. On the other hand like our case the 
sensor box has a temperature sensor with it. The gas mixing period helps to create mainly a homogenous temperature field within the chambers gas volume which showed a constant temperature throughout the measurement period. The meteorological data was gathered from

273 the university weather station [20], the average measured wind speeds were $5 \mathrm{~m} / \mathrm{s}$ on location.

274 The chamber sensor box measured in relation to time the following parameters: ambient 275 temperature inside the chamber was $16 \mathrm{C}^{\mathrm{o}}$ with a dew point of $10 \mathrm{C}^{\mathrm{o}}$ while relative humidity was $40 \%$. The importance of site description comes from the need to link climatic factors with onsite measurements, because soil biological metabolism is strongly influenced be temperature. Atmospheric concentrations of carbon dioxide as provided by [21] on the month of May 2015 were 401ppm.

\subsection{Permeability Calculation}

281 There are many ways to obtain soil permeability values; its importance comes as it is an essential input parameter that is needed for the numerical modelling. This parameter is an essential input for the Darcy equation to model gas flow within the soil layers. Here we derive a reliable method to find permeability values for a soil layer as will be shown in equation (1.5). From the soil texture side experimentally the location is characterized as having a loamy sand texture which can be located on Figure 9 with poor drainage with $80 \%$ sand, $15 \%$ silt and 5\% clay. Finding the location intrinsic permeability is based on the model of porous material made up of parallel tubes of uniform sizes stated in [22] as shown in equation (1.0):

$$
\mathrm{K}=\frac{\theta}{2 \pi} \mathrm{D}_{\text {total }}
$$




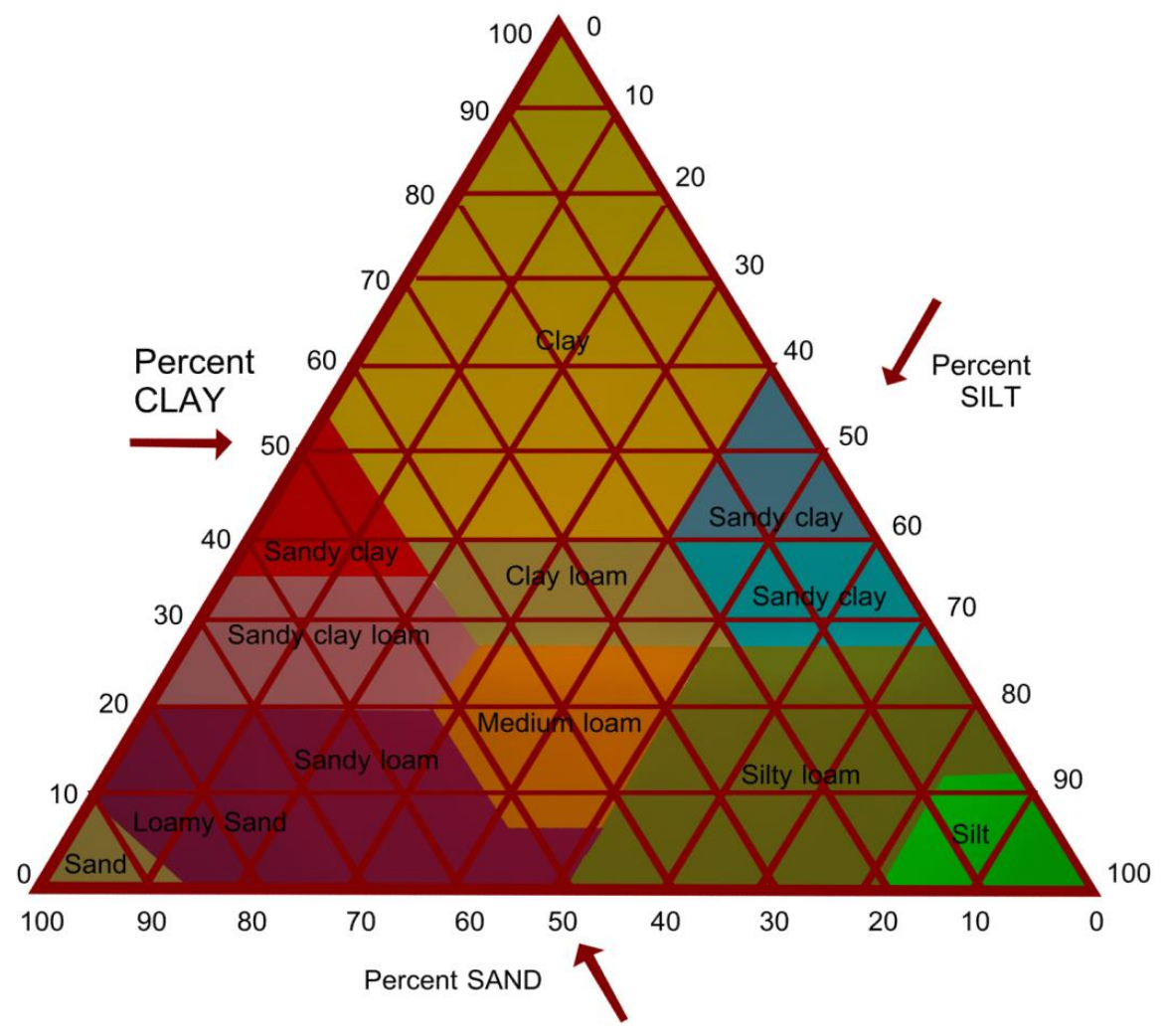

Figure 9: USDA and UK-ADAS Soil textural triangle.

292 To give the model the option of parallel tubes of different sizes pore size distribution is 293 characterized using a histogram, this is later applied to equation (1.0). The area of a single pore 294 area considered to have a circular area can be found using equation (1.1), where $d_{a v}$ is the 295 average pore diameter for a segment of pore sizes:

$$
\mathrm{A}_{\mathrm{n}}=0.25 \pi \mathrm{d}_{\mathrm{av}}^{2}
$$

296 Applying Newton's forward Integration law formula for two points on a single segment of the 297 histogram, where the two points represent the minimum and maximum value of pore diameters 298 at that segment:

$$
D_{n}=\int_{A_{n}}^{A_{n+1}} r(A) d A=\left(A_{n+1}-A_{n}\right) \frac{r_{n}}{2}
$$

299 The parameter $r$ is the mass ratio for one of the constituents found in the soil texture triangle 300 (example ratio of sand over total sum of constituents of sand silt and clay). By substituting 
equation (1.1) into (1.2) the area for a segment of pore size can be calculated using equation

$$
D_{n}=\pi\left(d_{n+1}^{2}-d_{n}^{2}\right) \frac{r_{n}}{8}
$$

303 Equation (1.3) can be extended to different size distribution by considering macro, meso and 304 micro pores, which for simplicity can be related to the ratios of sand, silt and clay:

$$
K=\frac{\theta}{2 \pi} \sum_{1}^{\mathrm{n}=3} \mathrm{D}_{\mathrm{n}}=\frac{\theta}{2 \pi}\left(\mathrm{D}_{\text {sand }}+\mathrm{D}_{\text {silt }}+\mathrm{D}_{\text {clay }}\right)
$$

305 Considering the pores diameters in equation (1.4) to be as follows for a top soil layer 306 macropores $(20-2000 \mu \mathrm{m})$, mesopores $(2-20 \mu \mathrm{m})$ and micropores $(0.2-20 \mu \mathrm{m})$ :

$$
\begin{aligned}
& \mathrm{K}=\frac{\theta}{2 \pi} \sum_{1}^{\mathrm{n}=3} \mathrm{D}_{\mathrm{n}}=0.25 \theta\left(\left(\mathrm{d}_{2}^{2}-\mathrm{d}_{1}^{2}\right) \mathrm{r}_{\text {sand }}+\left(\mathrm{d}_{3}^{2}-\mathrm{d}_{2}^{2}\right) \mathrm{r}_{\text {silt }}\right. \\
&\left.+\left(\mathrm{d}_{4}^{2}-\mathrm{d}_{3}^{2}\right) \mathrm{r}_{\text {clay }}\right)
\end{aligned}
$$

307 In conclusion the final form takes the form shown in equation (1.6):

$$
\mathrm{K}=\frac{\theta}{2 \pi} \sum_{1}^{\mathrm{n}=3} \mathrm{D}_{\mathrm{n}}=0.25 \theta\left(\mathrm{a}_{1} \mathrm{r}_{\text {sand }}+\mathrm{a}_{2} \mathrm{r}_{\text {silt }}+\mathrm{a}_{3} \mathrm{r}_{\text {clay }}\right)
$$

308 Where $\mathrm{a}_{1}=10^{-6}, \mathrm{a}_{2}=10^{-10}, \mathrm{a}_{3}=10^{-12}$. The power of this equation is that it allows the 309 researcher to get reasonable permeability values to be used for the simulation input based on firstly calcifying the site soil type. Consequently to later extract the ratios of sand, silt and clay from the soil texture triangle shown on Figure 9. This equation is only used to calculate 312 permeability using the soil texture triangle. Then the researcher can find soil porosity from available literature which can assist in finding the locations water content. Depending on the soil type located on the texture triangle you can find each soil texture has different storage capacity for water according to the ratios of sand, silt and clay. These ratios have already been 
found by scientists for grass land locations. Soil porosity for a loamy sand location (Grass land)

317 is in the range of 0.45 to 0.47 as reported by [23] .

318

319

320

321

322

323

324

326

327

\section{4 The K-Epsilon Turbulence Model}

To conduct the flow simulations ANSYS-CFX commercial software was used. The used turbulence model is the RANS model which is sometimes called K-Epsilon model [24]. The model is applied to the gas domain in the chamber, to resolve the occurring scalar field inside the chamber in relation to time. The turbulent kinetic energy $\mathrm{K}$ is defined as the variance of the fluctuations in velocity. This is followed by $\varepsilon$ which is the turbulence Eddy dissipation, which has a dimensions of $\mathrm{K}$ per unit time; for example. The K-Epsilon model introduces two new variables into the system of equations. The continuity equation is equation (1.7) where $\rho$ is the air density and $\mathbf{U}$ is the velocity vector field:

$$
\frac{\partial \rho}{\partial t}+\nabla \cdot(\rho \mathbf{U})=0
$$

The general momentum equations are:

$$
\frac{\partial \rho \mathbf{U}}{\partial \mathrm{t}}+\nabla \cdot(\rho \mathbf{U} \otimes \mathbf{U})-\nabla \cdot\left(\mu_{\mathrm{eff}} \nabla \mathbf{U}\right)=-\nabla \grave{p}+\nabla \cdot\left(\mu_{\mathrm{eff}} \nabla \mathbf{U}\right)^{\mathrm{T}}+\mathbf{B}
$$

Where $\mathbf{B}$ is the sum of body forces, $\mu_{\text {eff }}$ is the effective viscosity accounting for turbulence, and $\mathrm{p}$ is the modified pressure as defined in the following equation:

$$
\grave{\mathrm{p}}=\mathrm{p}+\frac{2}{3} \rho \mathrm{k}+\frac{2}{3} \mu_{\mathrm{t}} \bar{\nabla} \mathbf{U}
$$

The model is based on the Eddy viscosity concept, so that:

$$
\mu_{\mathrm{eff}}=\mu+\mu_{\mathrm{t}}
$$

where $\mu_{\mathrm{t}}$ is the turbulnce viscosity. The K-Epsilon model assumes that the turbulence viscosity is linked to the turbulence kinetic energy and dissipation via the relation: 


$$
\mu_{\mathrm{t}}=\mathrm{C}_{\mu} \rho \frac{\mathrm{k}^{2}}{\varepsilon}
$$

333 The parameter $\mathrm{C}_{\mu}=0.09$ is $\mathrm{k}-\varepsilon$ turbulence model constant. The values of $\mathrm{k}$ and $\varepsilon$ come 334 directly from the differential transport equations for the turbulence kinetic energy and 335 dissipation rate:

$$
\frac{\partial \rho \mathrm{k}}{\partial \mathrm{t}}+\nabla \cdot(\rho \mathbf{U k})=\nabla \cdot\left(\left(\mu+\frac{\mu_{\mathrm{t}}}{\sigma_{\mathrm{k}}}\right) \nabla \mathrm{k}\right)+\mathrm{P}_{\mathrm{k}}-\rho \varepsilon
$$

336 In addition

$$
\frac{\partial \rho \varepsilon}{\partial \mathrm{t}}+\nabla \cdot(\rho \mathbf{U} \varepsilon)=\nabla \cdot\left(\left(\mu+\frac{\mu_{\mathrm{t}}}{\sigma_{\varepsilon}}\right) \nabla \varepsilon\right)+\frac{\varepsilon}{\mathrm{k}}\left(\mathrm{C}_{\varepsilon 1} \mathrm{P}_{\mathrm{k}}-\mathrm{C}_{\varepsilon 2} \rho \varepsilon\right)
$$

337 Model used constants for (1.12) and (1.13) are taken as $\mathrm{C}_{\varepsilon 1}=1.44$ is, $\mathrm{C}_{\varepsilon 2}=1.92, \sigma_{\mathrm{k}}=1$ and 338 $\sigma_{\varepsilon}=1.3$.Turbulence production $\mathrm{P}_{\mathrm{k}}$ is the due to viscous and buoyancy forces, which are 339 modelled using equation (1.14):

$$
\mathrm{P}_{\mathrm{k}}=\mu_{\mathrm{t}} \nabla \mathbf{U} \cdot\left(\nabla \mathbf{U}+\nabla \mathbf{U}^{T}\right)-\frac{\mathbf{2}}{\mathbf{3}} \nabla \cdot \mathbf{U}\left(3 \mu_{\mathrm{t}} \nabla \cdot \mathbf{U}+\rho \mathrm{k}\right)+\mathrm{P}_{\mathrm{kb}}
$$

340 The average flow velocity encountered in the chamber is $2.7 \mathrm{~m} / \mathrm{s}$ subsequently the flow 341 simulation case is an incompressible flow one. Hence $\nabla . \mathbf{U}$ is small and the second term on the right side of equation (1.14) does not contribute significantly to the production term. Therefore, this leads to the conclusion that there is no need to use a more sophisticated turbulence model such as LES. It is commonly known that the mentioned model requires additional computational resources to be allocated to run the calculation. The aim of using CFD 346 simulations here is mainly to capture mass transport within the chamber and soil domain, it is not of priority to capture turbulence structures because a rotating fan wheel is not considered here. 
The Darcy model is derived from Reynolds transport theorem when applied to porous media.

351 This is achieved mainly by considering pressure as scalar quantity in the Reynolds transport 352 theorem. Gas exchange occurs in the studied simulation between the soil and gas medium, 353 meaning that we can rely on the theory of air movement due to pressure fluctuations [25].

354 Available in the ANSYS-CFX solver is the porous model which is at once both a generalization 355 of the Navier-Stokes equations and of Darcy's law. The main advantage of using commercial software is that they come with efficient mesh generation algorithms and tools giving the user the advantage of using the CFD code on complex geometries. The Darcy model [24] retains both advection and diffusion terms and can therefore be used for flows in the soil domain where such effects are important. In deriving the continuum equations, it is assumed that 360 'infinitesimal' control volumes and surfaces are large relative to the interstitial spacing of the porous medium, but small relative to the scales that wish to resolve. Thus, from the generated mesh the given control cells and control surfaces are assumed to contain both solid and fluid regions. The volume porosity $\gamma$ at a point is the ratio of the volume $\mathrm{V}^{\prime}$ available to flow in an infinitesimal control cell surrounding the point, and the physical volume of the cell. Hence:

$$
\mathrm{V}^{\prime}=\gamma \mathrm{V}
$$

It is assumed that the vector area A available to flow through an infinitesimal planar control 366 surface $A^{\prime}$ is given by equation $(1.16)$ where $K=\left(\mathrm{K}^{\mathrm{ij}}\right)$ is called the area porosity tensor:

$$
\mathrm{A}^{\prime}=\mathrm{K} . \mathrm{A}
$$

367 The dot product of a symmetric rank two tensor with a vector is:

$$
\mathrm{K} \cdot \mathrm{A}^{\mathrm{i}}=\mathrm{K}^{\mathrm{ij}} \mathrm{A}_{\mathrm{j}}
$$

ANSYS CFX presently only allows $\mathrm{K}$ to be isotropic. The general scalar advection-diffusion equation in a porous medium becomes: 


$$
\frac{\partial \gamma \rho \boldsymbol{\phi}}{\partial \mathrm{t}}+\nabla \cdot(\rho \mathbf{K} . \mathbf{U} \boldsymbol{\phi})-\nabla \cdot(\Gamma \mathrm{K} . \nabla \boldsymbol{\phi})=\gamma \mathrm{S}
$$
mass:

$$
\frac{\partial \gamma \rho}{\partial \mathrm{t}}+\nabla \cdot(\rho \mathrm{K} \cdot \mathbf{U})=0
$$

373 And momentum is:

$$
\frac{\partial \gamma \rho \mathbf{U}}{\partial \mathrm{t}}+\nabla \cdot(\rho \mathbf{K} \cdot \mathbf{U} \otimes \mathbf{U})-\nabla \cdot\left(\mu_{\mathrm{e}} \mathrm{K} \cdot\left(\nabla \mathbf{U}+(\nabla \mathbf{U})^{\mathrm{T}}\right)=-\gamma \mathrm{R} \cdot \mathbf{U}+\gamma \nabla \mathrm{p}\right.
$$

374 where $\mathbf{U}$ is the true velocity, $\mu_{\mathrm{e}}$ is the effective viscosity-either the laminar viscosity or a turbulent quantity, and $\mathrm{R}=\left(\mathrm{R}^{\mathrm{ij}}\right)$ represnts a resistance to flow in the porous medium. This is and diffusive terms on the left hand side are negligible. Hence equation (1.13) reduces to:

$$
\mathbf{U}=-\mathrm{R}^{-1} \cdot \nabla \mathrm{p}
$$

381 velocity:

$$
\mathbf{Q}=\mathbf{K} \cdot \mathbf{U}
$$

Subsequantly in the limit of large resistance, we obtain an anisotropic version of Darcy's law, with the permeability kept proportional to the inverse of the resistance tensor. However, unlike Darcy's law, we are working with the actual fluid velocity components $\mathbf{U}$, which are discontinous at discontinuity in porosity, rather than the continuous averaged superfical
Heat transfer is modeled with an equation of similar form: 


$$
\frac{\partial \gamma \rho \mathrm{H}}{\partial \mathrm{t}}+\nabla \cdot(\rho \mathbf{K} \cdot \mathbf{U H})-\nabla \cdot\left(\Gamma_{\mathrm{e}} \mathbf{K} \cdot(\nabla \mathrm{H})=\gamma \mathrm{S}^{\mathrm{H}}\right.
$$

387 Where $\Gamma_{\mathrm{e}}$ is an effective thermal diffusivity and $\mathrm{S}^{\mathrm{H}}$ contains a heat source or sink to or from 388 the porous meduim. A generalized form of Darcy's law is given by

$$
-\frac{\partial \mathrm{p}}{\partial \mathrm{x}_{\mathrm{i}}}=\frac{\mu}{\mathrm{K}_{\text {perm }}} \mathrm{U}_{\mathrm{i}}+\mathrm{K}_{\text {loss }} \frac{\rho}{2}|\mathbf{U}| \mathrm{U}_{\mathrm{i}}
$$

389 Therefore $\mu$ is the dynamic viscosity, $K_{\text {perm }}$ is the permeability and $K_{\text {loss }}$ is the empirical loss 390 coefficient.

\section{Numerical Analysis}

392 This section is composed of the simulation setup, results and discussion.

\subsection{Simulation Setup}

394 The finite element model is composed of two domains; a porous domain representing the soil, and a gas domain representing the air Figure 10. The Navier-Stokes equations are solved to resolve the occurring flow pattern in the chamber. To model the turbulent nature of the flow, the K-Epsilon turbulence model (1.9-1.14) is applied to the Navier-Stokes equations (1.8). The Darcy equation (1.24) is solved in the porous domain to resolve the occurring flow in it. Both domains model multiple species which are air and carbon dioxide.
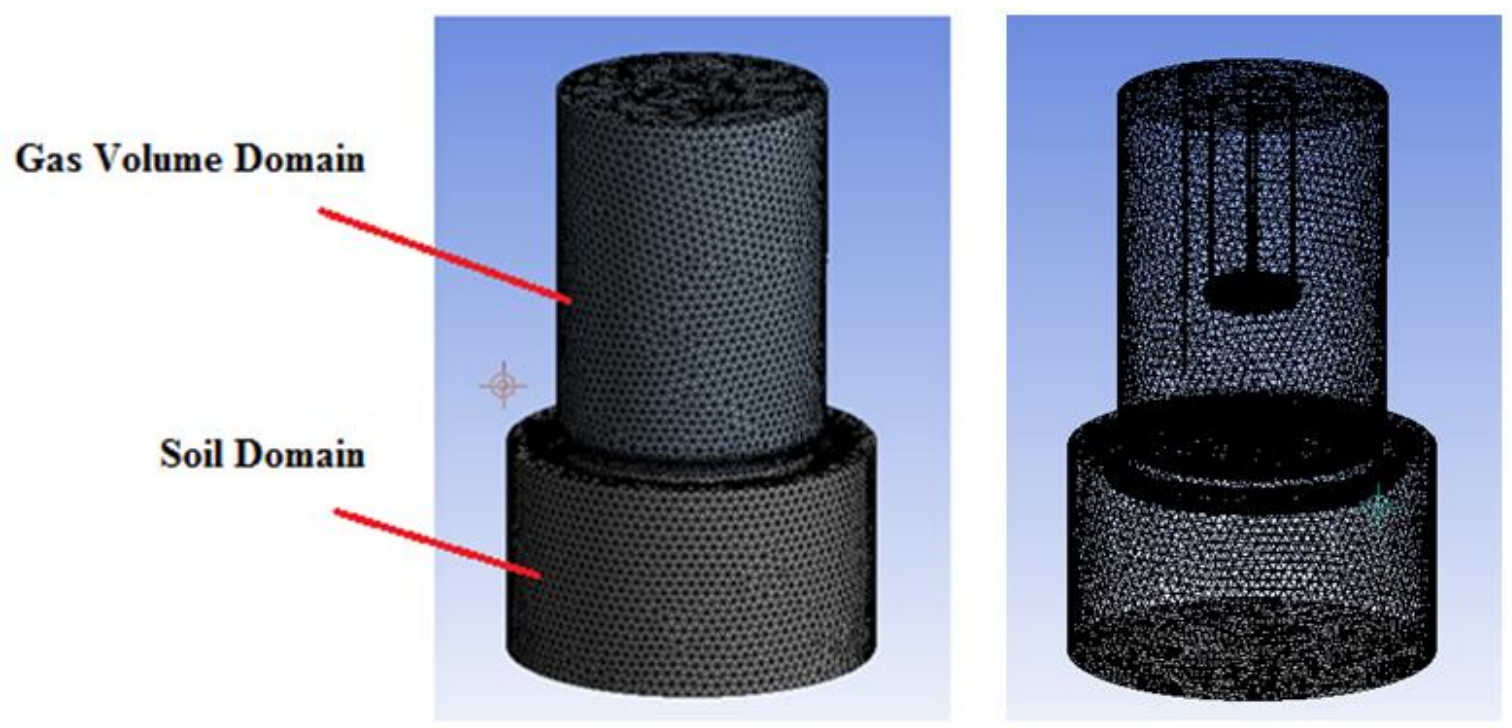
Figure 10: on the left the image shows the gas and porous soil domain moreover the right handside image shows the generated mesh quality for both the soil and gas volume domain

To solve the mentioned equations the two domains have to be broken up into discrete elements as shown on Figure 10. The tetrahedral mesh type is selected with medium size relevance centre this mesh generation algorithm generates a homogenous size of elements in both domains. Consequently this helps in providing volume elements that transport gas species at an instance of time uniformly from the bottom of the soil domain to the tip of the gas sensor located in the gas volume domain. Hence these cell volumes have a Peclet number greater than 1 for the gas domain. On the contrary for the soil domain they should have a Peclet number smaller than 1. The fan inflow and out flow boundary condition as shown in Figure 11 is set to $2.7 \mathrm{~m} / \mathrm{s}$ while all other surfaces are considered as wall boundary condition.

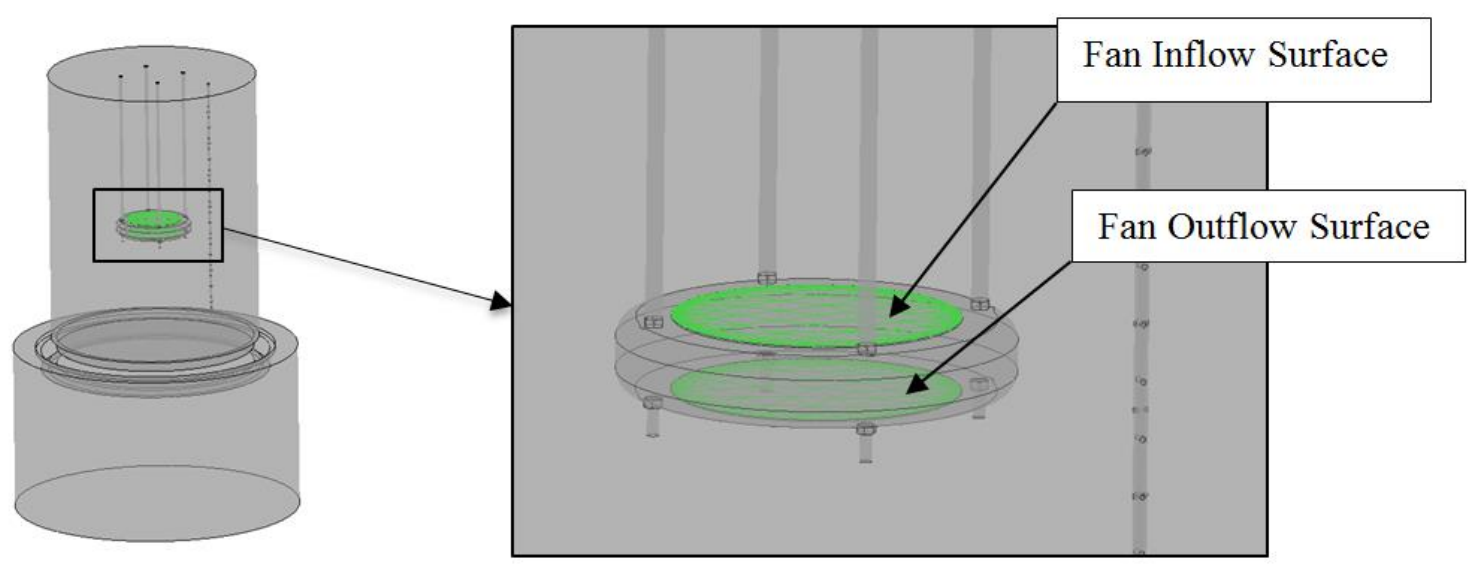

Figure 11: The inflow and out flow boundary surface for the fan location is highlighted in green.

There are two soil surfaces for the porous media as shown in Figure 12. One is located inside the chamber and the other outside the chamber. The top soil surface outside the chamber is assigned an atmospheric pressure boundary condition, while inside the chamber is assigned the interface boundary condition. 


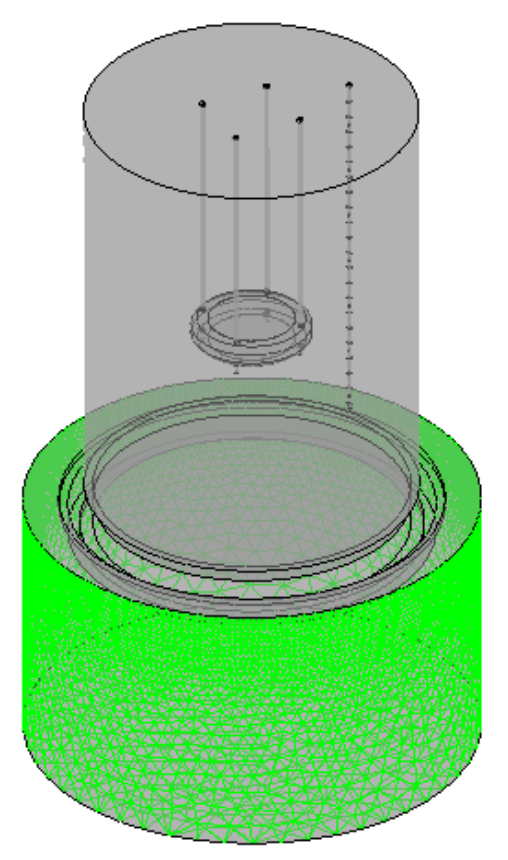

421

422 Figure 12: The side boundary surfaces that have been assigned an opening boundary

423 condition are laminated in light green.

424

425 The two domains are linked up using an interface boundary condition to model the mass

426 transport occurring between the both of them this is at the soil inside surface area inside

427 chamber shown in Figure 13. Gas exchange is modelled at the interface soil surface between

428 the soil and gas volume within the chamber as shown on the green surface in.

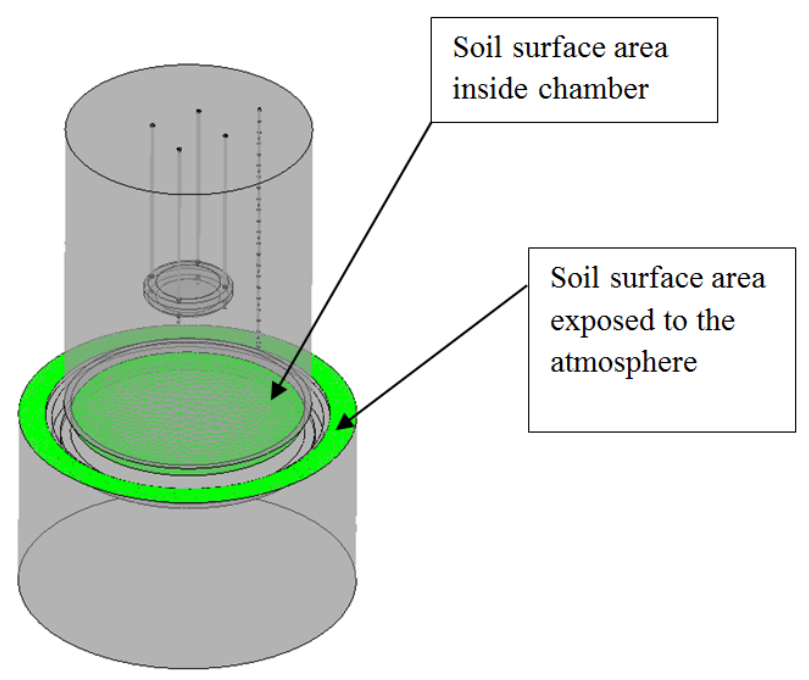


Figure 13: The highlighted boundary condition represents the interface boundary condition between the gas volume and soil porous domain furthermore represents the boundary of the porous domain with the atmosphere.

The carbon dioxide efflux is a result of biological activity in the soil and plants at the location.

To represent occurring biological activity without over complicating the project through going into its chemistry the bottom surface of the porous domain is assigned a carbon dioxide source term. The porous domain is assigned a 0.45 porosity based on [23]. The simulation permeability value is calculated from equation (1.6) after analysing the soil sample experimentally resulting $10^{-10} \mathrm{~m}^{2}$. Both atmospheric temperature and pressure are considered to be constant with time. Consequently atmospheric pressure is taken to be $1 \mathrm{~atm}$ and ambient temperature to be $16 \mathrm{C}^{\mathrm{o}}$. Time stepping is conducted using first order Euler method while a time step of $1 \mathrm{sec}$ is considered. The total simulation time is 360 seconds, this time period is generally enough to capture the gas species concentration jump which usually occurs the first 120 seconds. What happens after the saturation point is that any addition of gas species doesn't contribute to any addition of concentration jumps. In conclusion the concentration curve in relation to time after the point of saturation becomes asymptotic. This later contributes to the numerical model validation process with experimental data which as shown on Figure 16. The assigned initial condition is zero velocity with a volume fraction of one for Air. So that the simulation calculation starts with a pure air case for both the soil and gas domain. As the simulation progresses with time carbon dioxide species disperses gradually through the two domains. The simulation is run on a 16 GB RAM machine with a quad core Intel processer.

\subsection{Convergence check and mesh independence}

Looking at Figure 14 shows plots that are necessary to conduct a convergence check for the conducted numerical simulation this is done for a 100 time iteration, hence due to no evident jumps within the solution curves and to that they are in an order of $10^{-3}$ no further mesh dependence is required. Moreover the left plot shows the convergence of the solution for the 
rms velocity components in the $\mathrm{x} y$ and $\mathrm{z}$ axis. Likewise the same is conducted for the rms

457 pressure term. The three velocity components iterate at the same extent is due to the

458 homogenous vector filed created in the gas volume. The reason for the fluctuations with the

459 accumulated times steps is due to the changes of flow energy inside the chamber gas volume.

460 This is more evident on the right hand side for the convergence plot for the K-Epslion

461 turbulence model terms. Consequently the rms term for the kinetic energy term fluctuates and

462 the same applies for the epsilon term which is responsible for dissipating the flow kinetic

463 energy.

464 Furthermore commenting on Figure 15 shows a more constant iteration pattern for the rms

temperature term this is attributed to a steady heat transfer rate is occurring inside the chamber.

The evident noise in both rms enthalpy energy gas components is attributed to the dominance
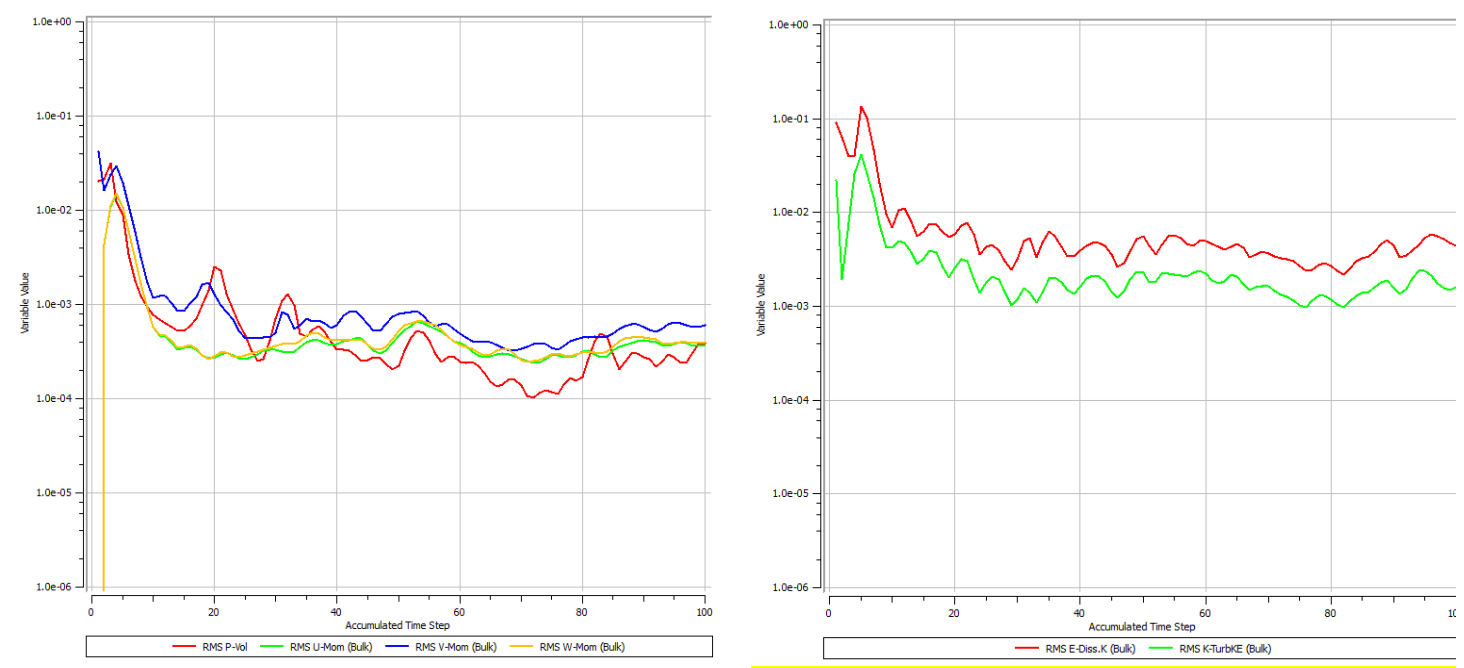

Figure 14: Convergence of the solution check in relation to accumulated time step, momentum and mass solution convergence on the left and the K-Epslion components used in the turbulence model on the right. 


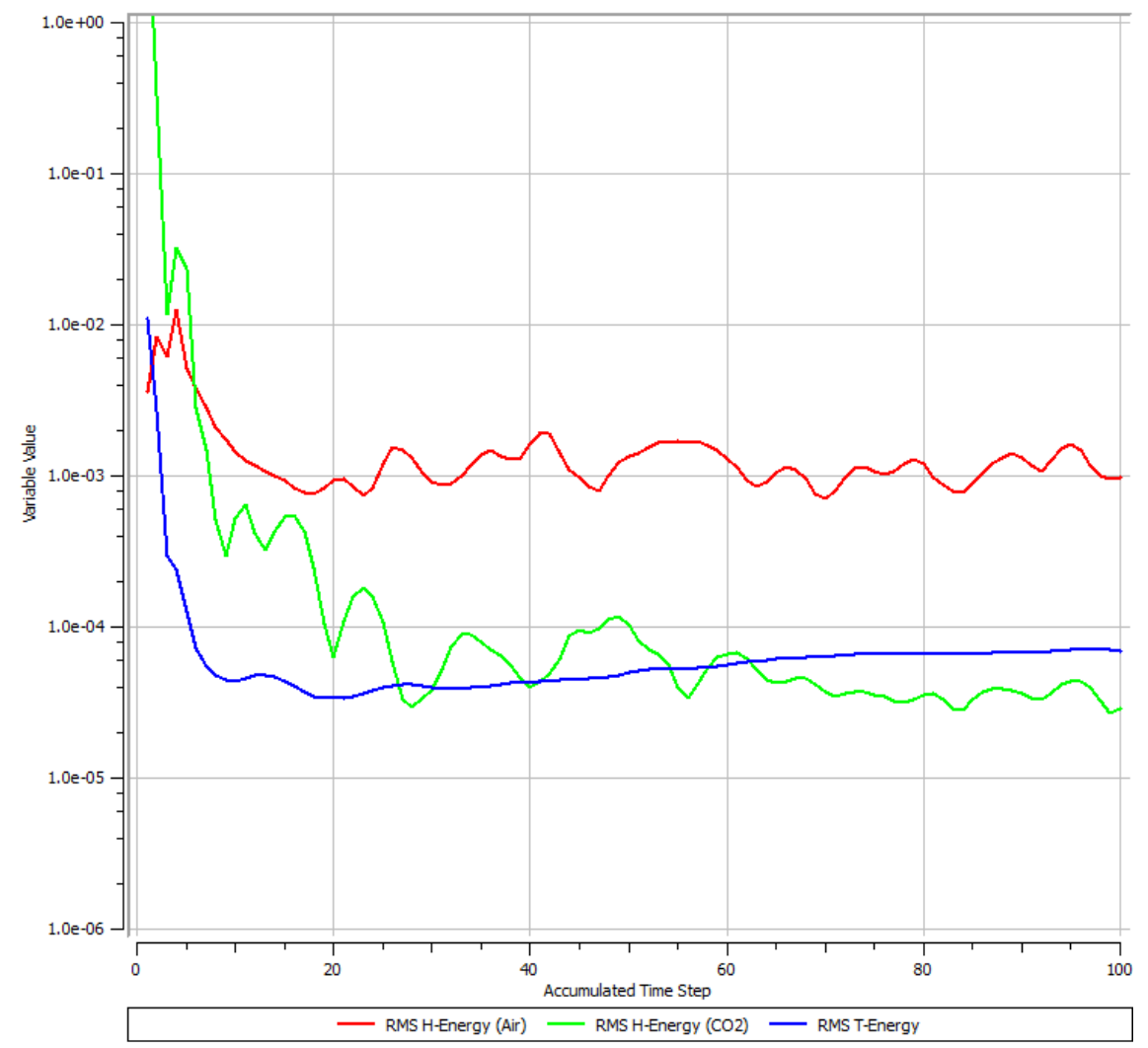

473 Figure 15: A convergence check of the solution for temperature in the energy equation 474 moreover the same check for both gases enthalpy convergence.

\subsection{Results and Discussion}

477 Figure 16 shows the experimental and flow simulation data obtained from the study. To examine the sensitivity and the frequency of the sensor sampling rate, samples initially were taken for two hours [26]. To conduct the carbon dioxide analysis, data from each 6 minute sampling period was manually analysed and the earliest 2-3 minute linear time period was used to calculate the carbon dioxide efflux in $\mathrm{g} / \mathrm{m}^{2} . \mathrm{h}$, based on equation (1.18):

$$
\overline{\mathrm{J}}_{\mathrm{g}}(\mathrm{t})=\frac{\mathrm{V}}{\mathrm{A}} \frac{\Delta \mathrm{C}}{\Delta \mathrm{t}}
$$

482 Where $\mathrm{V}$ is the chamber volume in $\mathrm{m}^{3}$ and $\mathrm{A}$ is the covered soil area in $\mathrm{m}^{2}, \Delta \mathrm{C} / \Delta \mathrm{t}$ represents 483 the carbon dioxide concentration derivative in relation to time mole $/ \mathrm{m}^{3} \mathrm{~h}$. The first 150 seconds time period is the necessary time to capture the flux jump that represents biological 
activity occurring on location. By using the developed MATLAB code at UWS and analysing the gathered data, this resulted in finding that the produced efflux on location is about $2 \mathrm{~mole} / \mathrm{m}^{2} \mathrm{~h}$ after 150 seconds of the experiment which is a reasonable value in relation to reported experimental data for a grass land location [27, 28] conducted using other types of chambers. Consequently a significant agreement between the experimental readings and numerical results was achieved meaning that CFD can be used to develop future respiration chamber designs as shown in Figure 16. Many measurements where conducted several days before the $7^{\text {th }}$ of May just to confirm repeatability of experiment and the obtained data. Speaking of Figure 16 it is visible from the CFD simulation, carbon dioxide concentration (subtracting $400 \mathrm{ppm}$ ) increases gradually with the progression of time till it becomes asymptotic with the experimental curve, noting that the asymptotic section of the data has been cropped out. No filtering function was applied for the gathered experimental data In Figure 16 because dynamic chambers generate turbulence within especially for our case whereby the gas sensor is plugged directly onto the chamber. From another perspective the measured fluctuations are due to the interaction of the convective flow with the drilled holes in the sampling tube. The transient period usually has these fluctuations in concentration but are not an issue because this is due to the gas mixture reaching the sensor tip as a function of the chamber turbulence. Our concern is when the measured mixture becomes asymptotic, after this point, we have no concern anymore because the captured initial jump is the objective. These holes purpose is to allow the mixed air carbon dioxide gas mixture to be collected and guided to the sensor tip. While the numerical data in In Figure 16 shows no fluctuations this is attributed to turbulence model used whereby its dissipation term is dominant. In Figure 16, a gauge concentration rise (similar to gauge pressure) of $100 \mathrm{ppm}$ for carbon dioxide is evident during a time period of 6 minutes. The rigid limit of $100 \mathrm{ppm}$ is because we are dealing with a gauge concentration measurement of carbon dioxide. This is resulting from biological activity 
within the soil and from the grass cover on location. The absolute atmospheric concentration of carbon dioxide within the atmosphere can be considered as fixed and has the value of 400 ppm. Usually for example at a grass land soil location the gauge average measured concentration is in the range of $60 \mathrm{ppm}$ at the first 100 seconds of measurements.

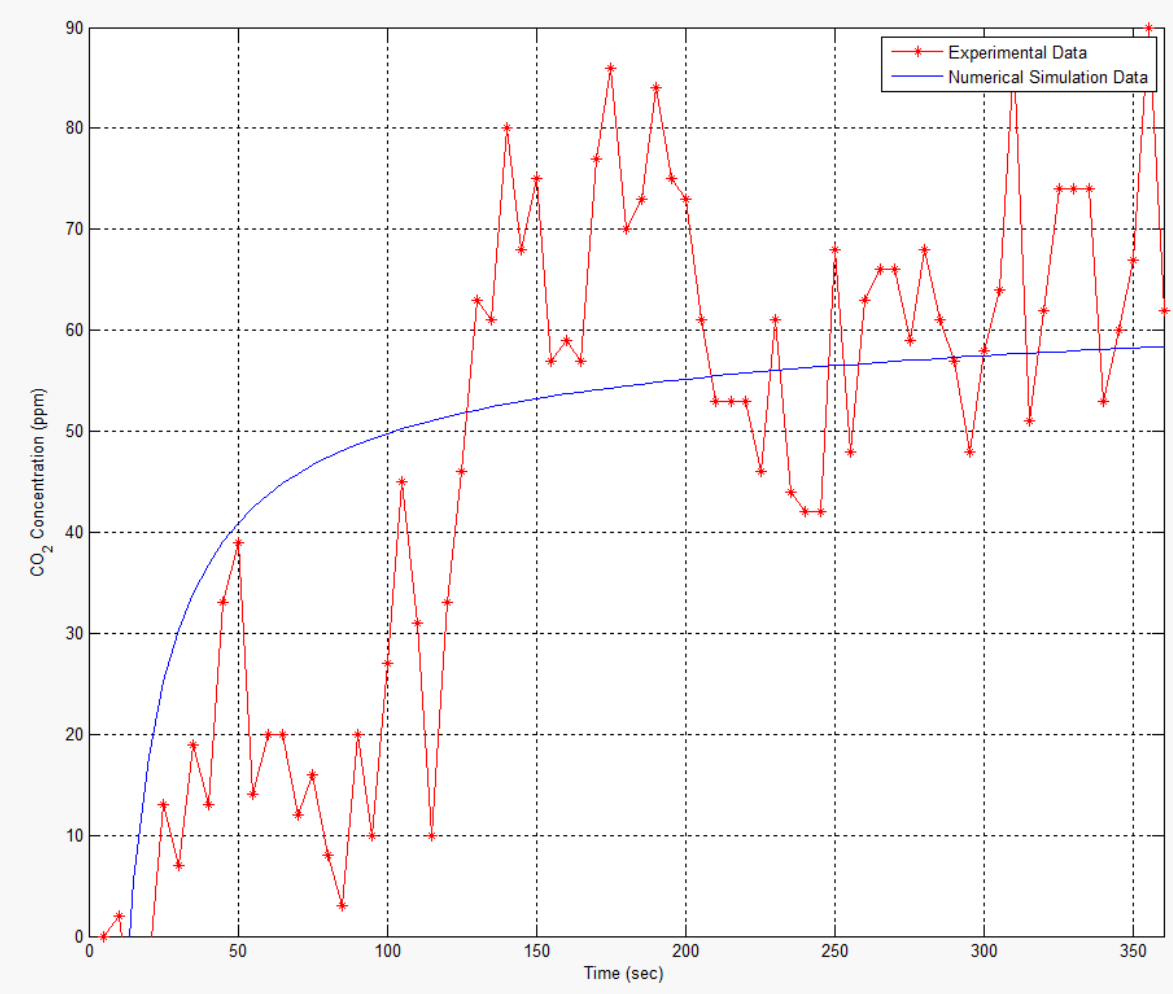

Figure 16: An experimental and numerical representation of carbon dioxide concentration with time inside the chamber.

For both curves a discrete increase in concentration exists in relation to time. The importance of this curve comes in validating the obtained results. The gas concentration data in the simulation is taken from the same location that the sensor is located in the experiment. This ensures that correct data values are measured in relation to time and space. Sensing location is of importance because sensed concentrations increase about $30 \mathrm{ppm}$ between two taken measurements as visible in Figure 16 for the case of experimental data at instance 80 and 90 sec. The deviations of measurement at every 5 seconds for the experimental data case occur due to convective flow occurring within the gas volume. They are attributed to the occurring 
turbulence in the dynamic run case. Commenting on the numerical simulation data the steady behaviour is due to application of the averaging method on the scalars of the Naiver Stokes equations hence that omits out measurement disturbance. In conclusion the numerical model shows the expected slope.

To view the process of capturing the grass land soil eflux experimentally and its challenges a static case is covered in Figure 17. It is common knowledge that the atmosphere is composed of many gases at different volume fractions. The measured atmospheric value of carbon dioxide concentration is $402.80 \mathrm{ppm}$ according to the authors in [29]. Hence to find gauge species concentration values inside the chamber atmospheric values of carbon dioxide where subtracted from the measurements. Consequently the gauge measured values where considered which start from zero to about $120 \mathrm{ppm}$ as shown in Figure 17. It is evident when looking at two plotted curves that in the top figure there is lots of disturbance to the measurements; therefore a MATLAB data filter is used at the bottom plot. The gauge concentration values are shown in the top figure while the bottom one shows the absolute concentration after applying a filtering function on it, noting that the filtered function is coloured blue furthermore the unfiltered one is in red. Classically researchers in literature use exponential function with one term to curve fit the disturbed set of data hence function (1.19) is obtained.

$$
C(t)=414 e^{\left(4 e^{-6}\right) t}
$$

543 The power of using the filtering function is that it provides the initial slop to measure the 544 biological soil activity. Consequently by multiplying the function in equation (1.19) by a filter $545 \mathrm{H}(\mathrm{z})$ the general formula can be found (1.20)

$$
\widetilde{\mathrm{C}}(\mathrm{t})=\mathrm{C}(\mathrm{t}) \mathrm{H}(\mathrm{z})
$$


546 A MATLAB built in function is applied on the gathered dataset from the experiment this is a 547 1-D digital filter [30]. This kind filter is used in signal analysis furthermore it can also be 548 applied to the collected data. A moving average filter is used and is represented by equation 549 (1.21):

$$
\mathrm{y}(\mathrm{n})=\frac{1}{\text { windowSize }}(\mathrm{x}(\mathrm{n})+\mathrm{x}(\mathrm{n}-1)+\cdots+\mathrm{x}(\mathrm{n}-(\text { windowSize }-1)))
$$

550 Hence the numerator coefficients of the rational transfer function are defined. For the studied 551 case it is taken as to have a value of windowSize $=30$. Moreover the denominator coefficients of the rational transfer function are taken to have the value of 1 . Filtering the rows or columns 553 of the efflux matrix with the following rational transfer function (1.22):

$$
\mathrm{H}(\mathrm{z})=\frac{1}{1-0.0333 \mathrm{Z}^{-1}}
$$

554 From Figure 17 by curve fitting the filtered function of concentration using an exponential 555 function with one term in relation to time equation (1.23) is obtained:

$$
\widetilde{C(t)}=391.8 \mathrm{e}^{\left(2.4 \mathrm{e}^{-5}\right) \mathrm{t}}
$$

556 What is evident when comparing equations (1.19) with (1.23) that both the filtered and 557 unfiltered function with a one term exponential function didn't capture the exact curve hence 558 the single term function was not considered. Furthermore an exponential function with two terms was adopted for curve fitting resulting in equation (1.24):

$$
\widetilde{C(t)}=420 \mathrm{e}^{\left(3.5 \mathrm{e}^{-6}\right) \mathrm{t}}-504 \mathrm{e}^{-0.0129 t}
$$

560 The extracted experimental equation is later used for the numerical simulation part of the 561 project whereby this obtained equation is read in into the ANSYS-CFX code. Consequently it 562 is assigned as a source term inside the biologically active soil. Without equation (1.24) it is very difficult to obtain correct results using CFD simulations. Both data sets are correct, one 

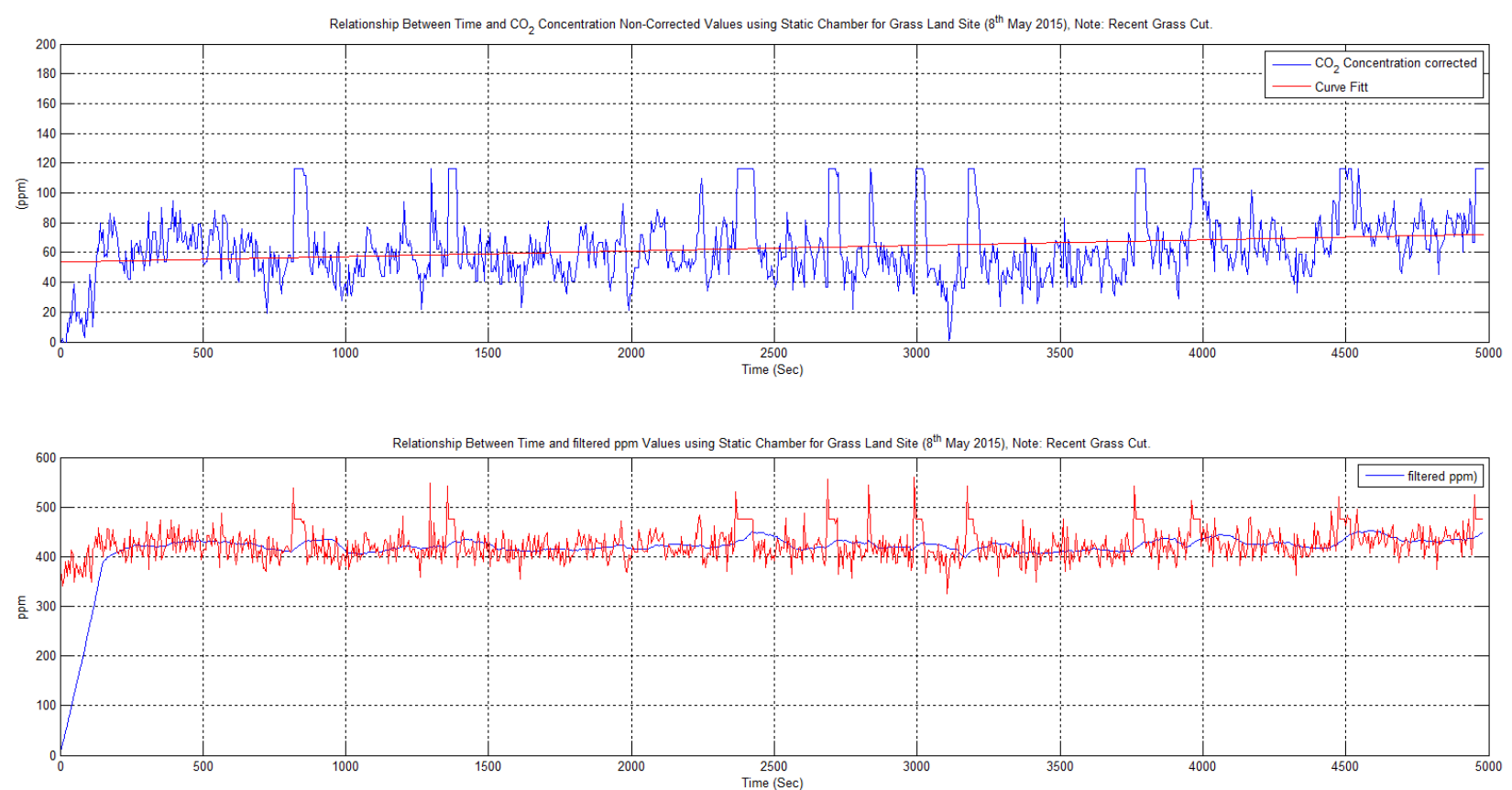

567 Figure 17: Guage concetration top curve, absolute concentration values of measured carbon

568 dixoide measuremnts bottom (red), filtered measurments of carbon dixoide (blue).

Speaking of Figure 18 shows the soil and fluid domains during the simulation for a dynamic chamber. The soil domain is taken down to a depth of $25 \mathrm{~cm}$ to ensure that all the necessary

572 flow details are captured in the simulation. Looking at the chamber gas domain, the visible

573 streamlines represent the flow velocity field starting from the blowing fan outlet boundary 574 which is facing the soil surface. 


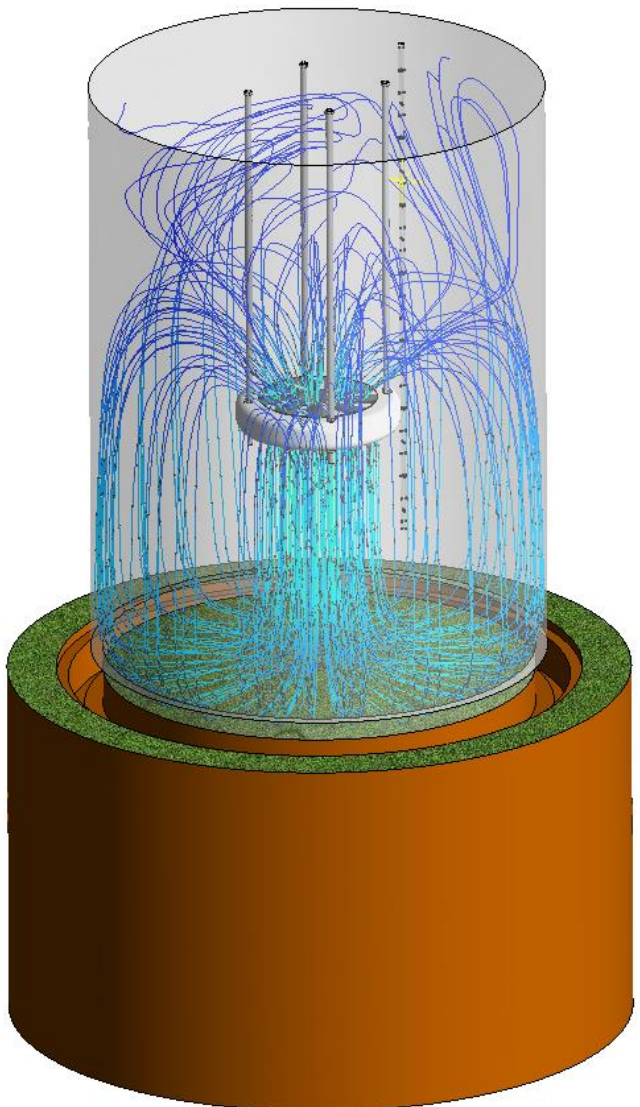

Figure 18: Capturing the occurring flow pattern in the studied chamber using stream lines.

578 These stream lines hits the soil surface and travel back on a parallel path to the chamber outer shell to be sucked into the fans inlet. Based on that the same mass inflow and outflow rate occurs for the fan it can be considered to have periodic boundary condition. The occurring circulation in the chamber is visible from the streamlines ensuring a fast homogenous mixture to be established in a short period of time. Consequently this is achieved by blowing air from a reasonable distance in relation to the soil surface. Hence this ensures the preservation of internal chamber pressure rise to a minimum. In conclusion carbon dioxide does not leak to the outside of the chamber. When the forced convective flow hits the soil surface it creates a wall shear stress. This wall shear stress produces a sucking effect that draws some of the soil carbon dioxide upwards instantaneously in the soil upper surface layers. The mixture is again sucked from the chambers headspace by the fan producing an internal periodic flow condition. The flow stream lines look symmetrical to the chambers main axis, the visible streamline 
590

591

592

593

594

595

596

597

598

599

600

601

602

603

604

605

606

607

608

609

610

611

612

613

irregularities are due to the applied turbulence model, this is mostly evident from the suction side of the fan with many twisted streamlines. Leaving the fan on for longer periods than 6 minutes can produce faulty fluxes due to the gradual build-up of internal pressure within the chambers gas volume. The 6 minute measurement time for a dynamic chamber case is enough to suck out all the stored carbon dioxide within the soil layers top layers.

\section{Conclusion}

The steps that pave the way to select and fit the right gas sensor at the right location within a respiration chamber have been covered in this paper. These steps start with specifying the design requirements and then proceeds to the $\mathrm{CAD}$ design stage. A portable device was designed, made and tested. It was proven that it can be used to measure accurately carbon dioxide concentration resulting from biological activity at specific locations of interest. To reduce the occurrence of wrong measurements the chamber sensor takes gas samples from all the elevations within the chamber using a sampling tube. Hence the sampling tube speeds up the gas sample going to the sensor tip this process over takes the diffusion time that is usually required for sensor measurement. This makes the sensor measurement frequency much higher in resolution in relation to time. The blowing fan fitting distance from the soil surface is selected thoroughly to draw out the carbon dioxide contained at the top soil biologically active layers. This is based on the design condition to preserve the chambers inner pressure which would contribute to any leakage out of the chamber. The researcher can to some extent rely on the air viscosity properties (Energy cascade theorem) to dampen the produced kinetic energy within the gas volume. CFD can contribute largely to the chamber development phase this in how to use it to model producing the right gas mixture ready for measurement. This is according to the experiment required sampling time, blowing fan speed and changing environmental parameters from temperature, pressure, etc. This works contribution is that it 
applied a numerical model on a gas volume and soil media to model gas exchange within a respiration chamber gas volume with a blowing fan that applies a jet flow onto the soil surface.

616 The objectives of this study were achieved by running numerical tests and then through 617 comparing them with the onsite measurements using the designed chamber on the grass land location. Validation of the produced data from the run simulations and apparatus showed the applicability of using such an apparatus for carbon dioxide efflux measurements. The results show that the K-Epslion turbulence model can be used to model flows in the closed dynamic respiration chambers. The developed numerical model can be applied to explore the occurring flow patterns for different chamber designs with different soil site locations. A MATLAB software can help in the data analysis stage of the project was developed. Consequently testing and calibrating new sensor technologies compatibility with recently developed chamber designs is applicable. The merits of using CFD tools include reductions in research costs and chamber development time. CFD can predict how homogenous is a gas mixture in the chamber gas volume also it can show mixing rates through visualizing the turbulence intensity and eddy frequency and flow strain rates within the chamber gas volume. Hence knowing the sensors frequency sampling rates and linking it with CFD can help in verifying if the sensors to be used within the chamber would perform their required role or not even before making full scale chamber. We can apply the different response function of concentrations for the sensor into the model. CFD has its limitations also and requirements this depends on the accuracy of the numerical methods used, furthermore on the generated calculation mesh and the size of the concentration in the order of $10^{-6}$ or $10^{-9}$. 


\section{Acknowledgments}

638 We would like to thank the lab technicians at UWS Institute of Engineering and Energy

639 Technologies, especially Mr Robert Boyce, for their support in the practical production stage.

640 Not forgetting Mr Tom Caddell for providing the meteorological site data from the university

641 on site weather station and technical assistance on PC hardware related issues. In addition to

642 that a big thanks to Dr Torsten Howind for his long talks relating to the field of geo-mechanics.

643

644

645

646

647

648

649

650

651

652

653

654

655

656

657

658

659

660

661

662

663

664

665

666

667

668

669

670

671

672

673 


\section{References}

675 1. Larkum, A.W.D., Contributions of Henrik Lundegårdh, in Discoveries in

2. Raich, J.W. and C.S. Potter, Global patterns of carbon dioxide emissions from soils.

Global Biogeochemical Cycles, 1995. 9(1): p. 23-36.

3. Bazzaz, F.A., The response of natural ecosystems to the rising global $\mathrm{CO} 2$ levels. Annual review of ecology and systematics, 1990: p. 167-196.

4. Joos, F., G. Müller-Fürstenberger, and G. Stephan, Correcting the carbon cycle representation: How important is it for the economics of climate change? Environmental Modeling \& Assessment, 1999. 4(2-3): p. 133-140.

5. Cramer, W., et al., Global response of terrestrial ecosystem structure and function to $\mathrm{CO} 2$ and climate change: results from six dynamic global vegetation models. Global Change Biology, 2001. 7(4): p. 357-373.

6. Parkinson, K., An improved method for measuring soil respiration in the field. Journal of Applied Ecology, 1981: p. 221-228.

7. Schlesinger, W.H. and J.A. Andrews, Soil respiration and the global carbon cycle. Biogeochemistry, 2000. 48(1): p. 7-20.

8. Widén, B. and A. Lindroth, A calibration system for soil carbon dioxide-efflux measurement chambers. Soil Science Society of America Journal, 2003. 67(1): p. 327334.

9. Roelle, P., et al., Measurement of nitrogen oxide emissions from an agricultural soil with a dynamic chamber system. Journal of Geophysical Research: Atmospheres (1984-2012), 1999. 104(D1): p. 1609-1619.

10. Kutsch, W.L., M. Bahn, and A. Heinemeyer, Soil carbon dynamics: an integrated methodology2009: Cambridge University Press.

11. Keith, H. and S. Wong, Measurement of soil CO 2 efflux using soda lime absorption: both quantitative and reliable. Soil Biology and Biochemistry, 2006. 38(5): p. 11211131.

12. Maier, M. and H. Schack-Kirchner, Using the gradient method to determine soil gas flux: A review. Agricultural and Forest Meteorology, 2014. 192: p. 78-95.

13. Aubinet, M., T. Vesala, and D. Papale, Eddy covariance: a practical guide to measurement and data analysis2012: Springer Science \& Business Media.

14. Janssens, I.A., et al., Assessing forest soil CO2 efflux: an in situ comparison of four techniques. Tree physiology, 2000. 20(1): p. 23-32.

15. Ngao, J., et al., Cross-calibration functions for soil $\mathrm{CO} \$_{-}\{2\} \$$ efflux measurement systems. Annals of forest science, 2006. 63(5): p. 477-484.

16. Lai, D., et al., The effect of atmospheric turbulence and chamber deployment period on autochamber $\mathrm{CO} 2$ and $\mathrm{CH} 4$ flux measurements in an ombrotrophic peatland. Biogeosciences, 2012. 9(8): p. 3305-3322.

17. Al Makky, A., et al., Renewable energy scenario and environmental aspects of soil emission measurements. Renewable and sustainable energy reviews, 2016.

18. Topographic Map Website. date of access 09/22/2015; Available from: http://engb.topographic-map.com/.

19. Lin, C.-J., et al., Empirical models for estimating mercury flux from soils. Environmental science \& technology, 2010. 44(22): p. 8522-8528.

20. University of West of Scotland Weather Station. Available from: http://weather.uws.ac.uk/.

21. CO2 Monthly. 2015; Available from: http://co2now.org/.

22. Marshall, T.J., J.W. Holmes, and C.W. Rose, Soil physics1996: Cambridge University Press. 
23. Koorevaar, P., G. Menelik, and C. Dirksen, Elements of soil physics. Vol. 13. 1983: Elsevier.

24. ANSYS, ANSYS CFX-Solver Modelling Guide, 2009.

25. Kimball, B. and E. Lemon, Theory of soil air movement due to pressure fluctuations. Agricultural meteorology, 1972. 9: p. 163-181.

26. Al Makky, A. et al. , A numerical and experimental study of a new design of closed dyanmic resperiation chambers. SEEP 2015: p. Pages 281- 286. State of the Art on Environmental Protection. SEEP 2015 Conference.

27. Pumpanen, J., et al., Comparison of different chamber techniques for measuring soil CO 2 efflux. Agricultural and Forest Meteorology, 2004. 123(3): p. 159-176.

28. Fierer, N., O.A. Chadwick, and S.E. Trumbore, Production of CO2 in soil profiles of a California annual grassland. Ecosystems, 2005. 8(4): p. 412-429.

29. CO2Now. 2016, date of access 6/1/2016; Available from: http://co2now.org/.

30. mathworks.1-D digital filter. Available from:

http://uk.mathworks.com/help/matlab/ref/filter.html. 